\title{
EL VALOR DE LA SOSTENIBILIDAD COMO FACTOR DE DIFERENCIACIÓN EN LOS PROCESOS DE INTERMEDIACIÓN TURÍSTICA: UN ANÁLISIS PARA EL CASO DE LAS PYMES CATALANAS
}

\author{
Lluís Garay \\ Joan Miquel Gomis \\ Francesc González. \\ Universitat Oberta de Catalunya
}

\section{RESUMEN}

La investigación sobre la sostenibilidad en el sector turístico se ha centrado en los últimos años en las grandes empresas de alojamiento. Aunque existen trabajos que analizan el impacto de la implementación de la sostenibilidad en los touroperadores, no se ha analizado suficientemente a las pequeñas y medianas agencias de viaje, que constituyen una parte importante de la estructura del sector. A través de una muestra cercana a las 120 empresas, el estudio demuestra la importancia de las motivaciones económicas pero también de los factores personales y la legitimización como motivos para configurar diferentes perfiles de agencias cuyo comportamiento sostenible difiere.

Palabras clave: Agencias de Viaje; Pequeñas y Medianas Empresas; Business Case; Altruismo; Estilo de vida; Legitimización.

The value of sustainability as a factor of differentiation in tourism intermediation processes: an analysis of the catalan smes

\section{ABSTRACT}

Research on sustainability in the tourism sector has been focused in recent years on large accommodation companies. Although diverse studies analyse the impact of sustainability

Recibido: 22 de abril de 2016

Devuelto para su revisión: 21 de noviembre de 2016

Aceptado: 13 de enero de 2017

Estudios de Economía y Empresa. Universitat Oberta de Catalunya. Avenida Tibidabo, 39, 08035, BARCELONA (España).E-mail: lgaray@uoc.edu,jgomis1@uoc.edu, fgonzalezre@uoc.edu 
in large tour-operators, this topic has not been sufficiently analysed in the case of small and medium-sized agencies, which are an important part of this sector's structure. Through a sample of nearly 120 companies, this study demonstrates the importance of economic motivations but also personal and legitimizing drivers to conform three different intermediaries' profiles whose sustainable behaviour differs.

Keywords: Travel Agencies; Small and Medium Enterprises; Business Case; Altruism; Lifestyle; Legitimization.

\section{INTRODUCCIÓN}

El análisis de la implementación de la sostenibilidad en el sector turístico ha avanzado en los últimos años, especialmente centrado en la consecución de mejoras en el negocio (business case) y focalizado especialmente en las grandes empresas del subsector del alojamiento. Cuando la literatura relacionada con la sostenibilidad ha hecho referencia a un subsector tan relevante como el de la intermediación, se ha centrado especialmente en los grandes touroperadores internacionales, olvidando a las agencias minoristas independientes. Este estudio intenta subsanar este olvido, puesto que de la misma forma que ocurre en el subsector del alojamiento, este tipo de empresas minoristas no solo son básicas en la estructura sectorial de la mayor parte de destinos internacionales sino que además también están protagonizando las mayores transformaciones en el sector, ya sea la relacionada con los procesos de des-intermediación y re-intermediación vinculados a la emergencia de Internet (Standing, Tang-Taye et al., 2014) como también la vinculada a la sostenibilidad y que adolece de un análisis pormenorizado. Precisamente el objetivo de este estudio es el de intentar obtener un perfil más definido de este tipo de agencias minoristas independientes en un momento de grandes cambios, observando las principales características de sus propietarios y directivos, sus propias características, y obviamente su comportamiento sostenible, al que consideramos especialmente definitorio de dichos cambios.

\section{REVISIÓN DE LA LITERATURA}

\subsection{La sostenibilidad en la empresa turística}

Aunque la literatura en el ámbito turístico (Bramwell y Lane, 1993) retrotrajo los orígenes teóricos del concepto de sostenibilidad turística a inicios de los años setenta, no fue en realidad hasta los años ochenta cuando realmente despegó el concepto, en línea con la ideología proyectada por el conocido Informe Brutland (WCED, 1987). Éste establecía un nuevo paradigma de desarrollo, el de la sostenibilidad, fundamentado en tres principios también conocidos como pilares, que se pueden resumir de la siguiente manera: (1) continuar produciendo riquezas para satisfacer las necesidades de la población mundial (pilar económico), (2) velar por la reducción de las desigualdades entre los pueblos del mundo (pilar social) y (3) no degradar el medio ambiente que heredarán las generaciones futuras (pilar medioambiental). Dicho paradigma debía servir también de inspiración para 
el desarrollo de cualquier territorio o actividad, y evidentemente la actividad turística no quedó al margen. Así, se consideró que el paradigma podría ser una perfecta guía para mejorar los impactos positivos y minimizar los negativos del turismo y además, para poder tener éxito, este desarrollo turístico sostenible tenía que estar respaldado por una mayor disponibilidad y transparencia de la información así como con un mayor diálogo con los grupos de interés, partes interesadas o stakeholders (Mihalic, 2014), auténticos receptores y canalizadores de los citados impactos. Se suponía además la necesidad de disponer de un liderazgo político que hiciera posible esta transparencia y diálogo. Finalmente, este desarrollo por parte de la oferta debía estar en línea con las expectativas y satisfacción de la demanda.

A partir de los noventa, los análisis sobre la sostenibilidad en el sector proliferaron, moviéndose de una primera etapa donde primó la conceptualización y los aspectos teóricos, a otra donde los elementos prácticos fueron teniendo cada vez mayor importancia. En este mismo sentido Clarke (1997) añadía la idea de que el turismo sostenible debía ser un concepto en permanente evolución y sugería el hecho de alcanzar determinados objetivos, que también podían ser revisados a medida que se fueran alcanzando. En relación a los enfoques, aunque el interés por el impacto medioambiental se ha mantenido constante, aspectos como el cambio climático adquirieron más importancia a inicios del siglo XXI mientras que la perspectiva más de oferta y especialmente la ligada a la planificación, la estrategia y en general el management ha sido también más prolífica que la relacionada con la demanda (Ruhanen, Weiler et al., 2015). Además, autores Buckley (2012) entienden que en los próximos años elementos como la influencia de la ética y la relación de la paz y la prosperidad con la sostenibilidad pueden ser temas emergentes. De la misma forma, algunos estudios han realizado interesantes críticas al concepto. Por ejemplo, Hardy, Beeton et al. (2002) exponían también los riesgos de que el concepto de turismo sostenible se erosionara (como, indicaban, ya había ocurrido con el concepto de ecoturismo) debido a su posible identificación con prácticas publicitarias sin ningún resultado veraz y también observaban cierto sesgo a la observación de medidas e impactos en el eje medioambiental con poca atención al elemento comunitario. De la misma forma, para Weaver (2013) el turismo sostenible se sitúa en una difícil posición de equilibrio entre la propuesta capitalista del turismo masivo y la alternativa de un turismo que respete los fundamentos éticos.

En todo caso, es importante tener en cuenta que el debate en torno a la sostenibilidad en la actividad turística ha estado en los últimos años mucho más centrado en la oferta del destino turístico desde una perspectiva más macro que micro (Dwyer, 2005). En el análisis empresarial, y como la literatura ha tenido ocasión de demostrar, la prioridad por la mejora de los resultados económicos derivada de este nuevo paradigma de desarrollo ha centrado el debate sobre las motivaciones que inducen a introducir estrategias de sostenibilidad, especialmente desde la perspectiva de las grandes corporaciones (Salzmann, Ionescu-Somers et al., 2005; Carroll y Shabana, 2010). De hecho, como indica Mihalic (2014), parece que los agentes públicos han interiorizado más este paradigma que los privados. En este contexto de un sector empresarial turístico todavía poco alineado con los criterios de sostenibilidad (Buckley, 2012), se ha identificado no obstante un aumento de la relevancia de la sostenibilidad (Sheldon y Park, 2010) en sus negocios, en muchas 
ocasiones utilizando otro tipo de conceptos claramente relacionados, como en el caso del triple-bottom line (una alusión a la consecución de objetivos empresariales relacionados con los tres pilares) o aún con mayor asiduidad en el caso de la Responsabilidad Social Corporativa (RSC) (Coles, Fenclova et al., 2013).

Esta visión de la implementación de la sostenibilidad en las organizaciones turísticas (especialmente desde el enfoque de la RSC) ha sido ampliamente analizada (Lee y Park, 2009; Molina, Claver et al., 2009; Inoue y Lee, 2011), especialmente desde enfoques metodológicos cuantitativos que observan la posible relación entre las prácticas de sostenibilidad introducidas y los resultados financieros en las grandes empresas del sector. $\mathrm{Y}$ en gran medida esta literatura ha señalado que el comportamiento sostenible de las organizaciones no es incompatible con la obtención de beneficios económicos y que las actividades socialmente orientadas de las organizaciones turísticas aportan beneficios directos e indirectos a la sociedad (Nicolau, 2008).

Más allá de la predominancia por el análisis de las grandes empresas del sector, diversas referencias han estudiado también los motivos, barreras y efectos de la sostenibilidad en las pequeñas y medianas empresas (pymes) turísticas, organizaciones predominantes en el sector. En este caso, además de las metodologías cuantitativas (Garay y Font, 2012) han aparecido también enfoques cualitativos (Tzschentke, Kirk et al., 2008) que han enriquecido el análisis. Entre las principales motivaciones se encuentran aspectos como la mejora de los beneficios o la disminución de los costes (los elementos que se han relacionado bajo el paradigma del business case), pero también el altruismo o los aspectos ligados a la mejora de la reputación, mientras que las principales barreras identificadas son la falta de recursos y de conocimientos (Tzschentke, Kirk et al., 2008; Sheldon y Park, 2010; Barton, Schlenker et al., 2012; Garay y Font, 2012; Sampaio, Thomas et al., 2012; Font, Garay et al., 2016). Estos análisis han identificado también un creciente número de pymes turísticas que incorporan prácticas de sostenibilidad en sus organizaciones (Sheldon y Park, 2010), predominando las de carácter medioambiental por encima de las socioculturales, contemplando también si eran de carácter operativo o suponían cambios organizativos. Además, diferentes autores (Lee y Park, 2009; Inoue y Lee, 2011; Garay y Font, 2012; Font, Garay et al., 2016) han analizado las posibles relaciones entre las principales características de estas empresas y la introducción de estrategias de sostenibilidad, identificando relaciones significativas y positivas entre la implementación de alguna de estas prácticas y la percepción sobre la situación y evolución de los resultados financieros (Garay y Font, 2012). De la misma manera, Font, Garay et al. $(2014 ; 2016)$ han identificado diferentes clústeres de empresas configurados a partir de las motivaciones relacionadas con el sostenimiento económico del negocio así como el compromiso social y los aspectos ligados al estilo de vida, destacando este último en cuanto a la implementación de prácticas de sostenibilidad y un mayor compromiso con elementos como la confianza y la comunicación con los clientes.

La literatura parece mostrar como las motivaciones y comportamientos sostenibles pueden diferir entre las grandes empresas y las pymes turísticas. Estas últimas disponen de menos recursos financieros (Worthington, Ram et al., 2006) o de información acerca de los requerimientos del mercado y las oportunidades de cambio relacionadas con estas prácticas (Condon, 2004). Pero ante estas desventajas que podrían frenar la introducción 
de prácticas de sostenibilidad en estas organizaciones se identifican oportunidades para su desarrollo como su rápida y flexible capacidad de reacción ante determinados procesos y su estrecha vinculación con sus grupos de interés (Jenkins, 2009). Finalmente, aunque la toma de decisiones en este tipo de organizaciones suele venir determinada por la personalidad del propietario y sus valores (Perrini, 2006) según Nicolau (2008) también es cierto que los consumidores tienden a otorgar su confianza a organizaciones que como las observadas, suelen tener un mayor compromiso sostenible.

\subsection{La sostenibilidad en el subsector de la intermediación}

Distintos autores (Nicolau, 2008; Sheldon y Park, 2010) defienden que el análisis de la sostenibilidad en la industria turística debería abordarse desde la perspectiva de sus diferentes subsectores (hoteles, agencias de viaje, compañías aéreas) para entender sus características diferenciales. En todo caso, este tipo de análisis, más comunes en el ámbito del alojamiento, se han prodigado poco en otros sectores, con excepciones muy recientes como en el caso de la restauración (Alonso-Almeida, Bagur-Femenias et al., 2015). En el sector de la intermediación se han centrado ocasionalmente en los grandes tour operadores, prestando escasa atención al caso de las pyme (Sigala, 2008; Dodds y Kuehnel, 2010; Baddeley, Font et al., 2011). Un vacío de análisis que cabe cubrir ya que estamos hablando de un subsector de importancia central para la cadena de valor turística, al poner en relación de forma directa a los consumidores finales con los proveedores turísticos y por tanto también con los distintos componentes del destino (Poon, 2001; Del Alcázar, 2002; Millán y Esteban, 2002). El subsector de la intermediación goza de una información de valor estratégico sobre el comportamiento de los principales actores de la cadena de valor turística, que depositan su confianza en ellos, reduciendo el alto grado de incertidumbre que la organización y comercialización de viajes genera. Máxime en un entorno cada vez más cambiante en el que los grupos de interés locales deben participar activamente en la toma de decisiones estratégicas que afectan al desarrollo turístico de su territorio (Su, Wang et al., 2013).

Y ello en un contexto en el que observa por un lado una progresiva concentración de los grandes touroperadores (Vallespín Arán y Molinillo, 2014) mientras las agencias independientes de pequeña dimensión reposicionan sus propuestas de valor (AguiarQuintana, Moreno-Gil et al., 2016) a través de su presencia en Internet para reforzar sus factores diferenciales (entre ellos la sostenibilidad), convirtiendo a las TIC en un elemento básico de la comercialización turística (Bédard, 2003; Buhalis, 2003; Law, Leung et al., 2004; Kracht y Wang, 2010; Berne, Garcia-Gonzalez et al., 2012). De hecho, las empresas de intermediación turística son paradigmáticas en la transformación de modelos de negocio tradicionales provocada por la digitalización (Andal-Ancion, Cartwright et al., 2003; Gomis y González-Reverté, 2011). Y lo son porque desde la irrupción de internet en el mundo de los negocios las organizaciones dedicadas a la intermediación turística han ido viendo progresivamente alterado su rol en la cadena de valor. La tecnología ha abierto nuevos canales que facilitan la interactuación directa entre proveedores y consumidores finales alterando buena parte de las funciones que tradicionalmente éstas asumían (Borja y Gomis, 2009). En este entorno se han producido dos tipos de conse- 
cuencias con efectos transformadores en los procesos de intermediación. Por un lado, un acercamiento entre proveedores y consumidores finales, en detrimento de los agentes de viaje (Appelman, Go et al., 2001). Por otro lado, la aparición de nuevos operadores intermediadores y modelos de negocio diseñados a partir de organizaciones totalmente virtuales y estructuradas a partir de los sistemas de información (Chiou, Lin et al., 2011; Oroian y Gheres, 2012).

En este escenario se producen los procesos de re-intermediación en los que la función de intermediación redefine su función en la cadena de valor (Andal-Ancion, Cartwright et al., 2003; Borja y Gomis, 2009). En este contexto, el intermediario debe identificar y reivindicar el valor añadido que aporta tanto a proveedores como a consumidores y debe hacerlo en un entorno cada vez más competitivo, básicamente por dos factores: la voluntad de ahorro de costes en la distribución con la venta directa por parte de los proveedores y la aparición de nuevos intermediarios como las agencias online (Online Travel Agencies o OTA en su acrónimo en inglés), caso de Expedia o Travelocity; centrales de reserva hotelera, caso de Booking; y la expansión de las redes sociales y sus modelos de negocio asociados, incluidos los P2P, caso de Tripadvisor o AirBnB. Desde esta perspectiva es relevante la aportación de Ford, Wang y Vestal (2012) sobre el poder asimétrico que están otorgando las redes de distribución turística, a través de las cuales organizaciones teóricamente menos poderosas (como las pyme de este subsector) tienen ahora a su alcance estrategias que les pueden otorgar más valor en sus operaciones.

El análisis de la implementación del valor de la sostenibilidad en este subsector y en concreto para el caso de las pymes debe establecer si en este nuevo entorno impulsado por las tecnologías éstas tienen en la sostenibilidad una oportunidad para resultar más competitivas. En todo caso para que esta ventaja pase a ser sostenible en el tiempo es necesario que estos recursos no sean imitables sin gran esfuerzo por parte de la competencia y que realmente puedan ser identificados como únicos por parte del mercado (Inoue y Lee, 2011). De hecho, esta última posibilidad ha sido la que ha recibido más interés en los últimos años, especialmente a través de la denominada visión basada en los recursos y competencias (resource based view) (Inoue y Lee, 2011), que sugiere que la implementación de la sostenibilidad puede venir motivada por la posibilidad de originar recursos y competencias distintivos y valiosos que podrán proporcionar a la empresa una ventaja competitiva futura basada en la diferenciación (Hart, 1995).

Desde esta perspectiva, el presente artículo se propone analizar en qué medida las empresas de intermediación identifican la sostenibilidad como factor de diferenciación determinante en un entorno tecnológico, competitivo y complejo como en el que se mueve la comercialización turística. Se trata de cuantificar hasta qué punto la sostenibilidad puede representar una ventaja competitiva para una organización que la implementa, en relación a aspectos determinantes en los procesos de compra como el precio (Davies y Downward, 2007). En este sentido, se trataría de evaluar si las pymes del sector de la intermediación pueden identificar una ventaja competitiva frente a los grandes operadores del sector de la intermediación con capacidad de comercialización orientada al bajo coste. Consideramos que este análisis es relevante, dada la posición privilegiada que el intermediario ocupa en la cadena de valor turística para explorar la interacción entre los distintos actores de la cadena sobre su percepción de las obligaciones éticas y 
las normas. Como mediador, el intermediario debe considerar y conciliar los intereses del consumidor y las variables que condicionan sus procesos de decisión de compra; del proveedor y sus estrategias de rentabilidad; y del territorio de destino y la población local y su desarrollo sostenible desde el punto de vista medioambiental, social y económico (Waligo, Clarke et al., 2013). Y es que el intermediador se sitúa justo en el centro de las cadenas de valor de las estructuras de mercado turísticas, teniendo así su actuación una gran influencia para el resto de stakeholders o grupos de interés de éstas y por ende en el mismo territorio o destino, pudiendo influir también en la generación de territorios sostenibles (Clavé y Reverté, 2007).

\section{METODOLOGÍA}

\subsection{Población y muestra}

La investigación empírica se ha llevado a cabo durante 2014 y 2015 en Cataluña (España), donde el sector turístico representa uno de los principales motores económicos de la región, con un 11\% del PIB y cerca de 200.000 personas empleadas en este sector sobre todo en las pequeñas y medianas empresas (Idescat, 2014). Esta investigación supone un paso más en una serie de estudios realizados en los últimos años, enfocados en el análisis de la implementación de prácticas de sostenibilidad en la pyme turística, pero especialmente centrados en el alojamiento, dando lugar a una serie de publicaciones científicas al respecto (referencias omitidas). Con la presente investigación pretendemos subsanar la falta de estudios empíricos sobre la introducción de medidas de sostenibilidad en el sector de la intermediación, tanto por su relevancia como por el hecho que la sostenibilidad en sus pymes no ha sido suficientemente analizada.

La población de este estudio estaba compuesta por el total de agencias de viaje que opera en Cataluña, disponible en una base de datos proporcionada por el gobierno autonómico catalán (DEO, 2014) con 2.592 establecimientos, de los cuales 2.103 tenían un correo electrónico válido (con exclusión de los correos que no funcionaban y también casos en los que un solo mail se utilizó para varios establecimientos). El estudio se inició con un envío de nuestro cuestionario a una muestra soft de 150 empresas y cuando el método fue validado se realizaron tres rondas de recolección de datos durante un período de 6 semanas, en mayo-julio de 2014, justo a las puertas de la temporada alta turística. La muestra resultante de 119 establecimientos proporciona resultados válidos y fiables con un margen de error inferior al 9\%, un nivel de confianza mayor que el $95 \%$ y un nivel de heterogeneidad de $50 \%$ considerando los supuestos tradicionales de una distribución normal. El Alfa de Cronbach de las principales variables tuvo un resultado positivo y cercano a 1 , lo que sugiere que las diferencias entre las empresas participantes y no participantes no fueron estadísticamente significativas. La muestra y la población tienen porcentajes similares de empresas por su tamaño y el número de empleados (Idescat, 2014). Finalmente, se utilizaron medidas para garantizar la representatividad de la muestra, que dieron los resultados esperados. Entre éstas, el hecho de no encontrar importantes sesgos por deseabilidad social o una distribución de las empresas en el territorio similar a la de la población. 


\subsection{Metodología, variables y medidas}

Diversos meta-análisis como el de Coles, Fenclova et al. (2013) clarifican el hecho que la mayor parte de los estudios que relacionan la introducción de medidas de sostenibilidad con la obtención de mejoras en el negocio (especialmente financieras, en línea con el business case) han utilizado metodologías cuantitativas (Nicolau, 2008; Lee y Park, 2009; Garay y Font, 2012; Font, Garay et al., 2014). Y lo han hecho en muchos casos empleando variables y medidas que ya habían sido testadas en otros sectores o en todo caso, utilizando variables métricas dispares y de difícil estandarización. Precisamente Coles, Fenclova et al. (2013) indican que los aspectos relacionados con la métrica han sido los menos desarrollados en este tipo de literatura (ya sea en el sector turístico como en otros sectores). En todo caso, en este estudio se ha realizado un esfuerzo por recopilar un gran volumen de información relativa al tipo de variables y medidas utilizadas, a la par que una adaptación al contexto de la intermediación. En este sentido, cabe empezar aclarando que, siguiendo los objetivos del estudio y a partir de las referencias que se muestran en la tabla 1, el cuestionario que se envió a la población antes presentada preguntaba en primer lugar a los encuestados acerca de si mismos y posteriormente acerca de su negocio. A continuación, preguntaba acerca de la implementación de la sostenibilidad, teniendo en cuenta los motivos y barreras, así como las prácticas implementadas para finalmente preguntar sobre su opinión acerca de los resultados obtenidos a partir de la implementación de la sostenibilidad y sobre el futuro de esta estrategia.

Las variables relacionadas con las características del encuestado y del negocio son, como se puede observar, las habituales en este tipo de estudios y especialmente las del negocio se relacionan directamente con las principales características de las empresas del sector de la intermediación. Al respecto de las variables relacionadas con la sostenibilidad es importante resaltar que son fruto de una ya consistente literatura (como también se aprecia en la tabla 1) en esta materia, pero especialmente centrada en otros subsectores turísticos, especialmente el del alojamiento. En este sentido, la existencia de motivaciones de tipo altruista (ambientales, sociales, de estilo de vida), económicas (costes, imagen) y de legitimización con diversos grupos de interés, está ampliamente reportada, de la misma manera que el tipo de barreras (tiempo, recursos, desconfianza). Para el caso de las prácticas de sostenibilidad la base ha sido de nuevo la literatura relacionada con el alojamiento, aunque adaptándola a la realidad de la intermediación (y especialmente de su producto) y también en base a las recientes reflexiones en cuanto a la misma (Borja y Gomis, 2009; Vallespín Arán y Molinillo, 2014). Así, por ejemplo, cuando en un hotel se habla de los tradicionales ecosavings o ahorros en agua y/o energía, en nuestro estudio se incluyen a través de la promoción entre los clientes de este tipo de medidas. Y de la misma manera se ha llevado a cabo para otro tipo de medidas que van más allá de estas prácticas de carácter más operativo y que implican otro tipo de innovaciones y/o cambios en la organización. El mismo tipo de adaptación se ha efectuado en el caso de los resultados de la implementación de la sostenibilidad y las perspectivas de futuro a propósito de la sostenibilidad, donde también se ha tenido en cuenta tanto la literatura más reciente y destacada en relación a los impactos de la sostenibilidad en el sector turístico como las referencias que hacen mención al presente de la intermediación. 


\section{Tabla 1}

PRINCIPALES ASPECTOS, PREGUNTAS Y REFERENCIAS UTILIZADAS EN EL CUESTIONARIO

\begin{tabular}{|c|c|c|}
\hline Aspectos & Preguntas / Variables & Referencias \\
\hline $\begin{array}{c}\text { Perfil del } \\
\text { encuestado }\end{array}$ & $\begin{array}{l}\text { Sexo, Edad, Nivel de Estudios, } \\
\text { Rol (propietario, directivo) }\end{array}$ & $\begin{array}{l}\text { (Kim, Lehto et al., 2007; Carroll y } \\
\text { Shabana, 2010; Curtis, Conover et } \\
\text { al., 2012; Garay y Font, 2012; Piff, } \\
\text { Stancato et al., 2012) }\end{array}$ \\
\hline $\begin{array}{l}\text { Características } \\
\text { del negocio }\end{array}$ & $\begin{array}{c}\text { Antiguiedad del negocio, } \\
\text { Afiliación a alguna marca o } \\
\text { cadena, Empresas familiares, Tipo } \\
\text { de negocios, Categoría, Número } \\
\text { de empleados, Agencia mayorista } \\
\text { o minorista, Agencia Emisora o } \\
\text { receptora, Productos ofrecidos, } \\
\text { Destinos preferentes, Presencia } \\
\text { en las redes, Preferencias de los } \\
\text { clientes, Resultados financieros } \\
\text { presentes y evolución reciente }\end{array}$ & $\begin{array}{l}\text { (Appelman, Go et al., 2001; Poon, } \\
\text { 2001; Bédard, 2003; Condon, 2004; } \\
\text { Law, Leung et al., 2004; Salzmann, } \\
\text { Ionescu-Somers et al., 2005; } \\
\text { Tzschentke, Kirk et al., 2008; Borja y } \\
\text { Gomis, 2009; Jenkins, 2009; Dodds y } \\
\text { Kuehnel, 2010; Kracht y Wang, 2010; } \\
\text { Sheldon y Park, 2010; Baddeley, Font } \\
\text { et al., 2011; Chiou, Lin et al., 2011; } \\
\text { Inoue y Lee, 2011; Barton, Schlenker } \\
\text { et al., 2012; Berne, Garcia-Gonzalez et } \\
\text { al., 2012; Ford, Wang et al., 2012) }\end{array}$ \\
\hline $\begin{array}{l}\text { Características de } \\
\text { la sostenibilidad }\end{array}$ & $\begin{array}{c}\text { Motivaciones, Barreras, Prácticas } \\
\text { implementadas, Resultados } \\
\text { de la implementación de la } \\
\text { sostenibilidad, Perspectivas } \\
\text { de futuro a propósito de la } \\
\text { sostenibilidad }\end{array}$ & $\begin{array}{l}\text { (Markus y Kitayama, 1991; Kollmuss } \\
\text { y Agyeman, 2002; Morrow y } \\
\text { Rondinelli, 2002; Shane, Locke et } \\
\text { al., 2003; Condon, 2004; Salzmann, } \\
\text { Ionescu-Somers et al., 2005; Chan y } \\
\text { Wong, 2006; Kasim, 2007; Nicolau, } \\
\text { 2008; Tzschentke, Kirk et al., 2008; } \\
\text { Brønn y Vidaver-Cohen, 2009; } \\
\text { Jenkins, 2009; Carroll y Shabana, } \\
\text { 2010; Inoue y Lee, 2011; Barton, } \\
\text { Schlenker et al., 2012; Raviv, Becken } \\
\text { et al., 2013; Thapa y Best, 2013; Font, } \\
\text { Garay et al., forthcoming) }\end{array}$ \\
\hline
\end{tabular}

Fuente: elaboración propia.

Por lo que respecta a las medidas, cabe empezar diciendo que las características del encuestado y del negocio se han medido mediante variables dicotómicas. En estos casos la pregunta era simplemente si el encuestado o el negocio cumplían con determinada característica y no en que intensidad se podía valorar alguno de estos elementos. Lo mismo ocurre en relación a las prácticas de sostenibilidad, donde se ofrecía además los encuestados la posibilidad de seleccionar las que realmente tuvieran que ver con su comportamiento entre un amplio conjunto de posibilidades reportadas por la literatura 
que se muestra en la anterior tabla 1. Por otro lado, para los objetivos de este estudio no interesaba tanto observar cómo se implementaban las medidas, sino realizar una primera aproximación a cuáles eran efectivamente las prácticas de sostenibilidad que se estaban implementando. En relación a los motivos y barreras para introducir la sostenibilidad, así como a los resultados derivados o las perspectivas de futuro a propósito de la misma, se introdujeron escalas de Likert que midieran su intensidad y que posteriormente permitieran la posible conformación de factoriales como precisamente se demostrará en el apartado de resultados. Es importante añadir que se analizó la validez y fiabilidad de estas medidas. La validez de contenido se aseguró mediante una extensa revisión de la literatura así como a partir de la opinión de expertos de académicos y profesionales de la hostelería. La validez de cada constructo que se muestra en el apartado de resultados se evaluó a través de un análisis factorial para cada medida, al que posteriormente también se aplicó un análisis de fiabilidad que en todos los casos (factores creados para las variables de motivación, barreras y resultados) dio un Alfa de Cronbach mayor del 0,7 considerado necesario.

\section{RESULTADOS}

\subsection{Descripción de los encuestados y de los negocios}

Empezando por la descripción de los encuestados, podemos ver como ambos géneros están representados de manera casi equitativa ( $51,3 \%$ hombres y 48,7\% mujeres), con una media de edad de 46 años y un nivel educativo medio-alto. De estos encuestados, tan solo un $2,5 \%$ tiene solo estudios primarios y más de un $80 \%$ tiene estudios superiores. Los encuestados con estudios superiores son en un gran porcentaje diplomados en turismo (30,3\% de la muestra) pero también en otras titulaciones (37\% de la misma). La mayor parte de los encuestados son propietarios de las empresas $(62,2 \%)$ y el resto se distribuye de manera equitativa entre directores generales, de área y otros puestos. La muestra está compuesta por establecimientos relativamente jóvenes; aunque un 11\% tenga 30 o más años, casi un $60 \%$ tiene menos de 10 años de antigüedad. Se trata además de negocios independientes, donde solo un $11,8 \%$ está afiliado a una marca o franquicia, aunque la mayor parte $(54,6 \%)$ pertenezca a alguna asociación empresarial.

Al respecto de las características del negocio, en el Gráfico 1 podemos ver la localización de las empresas estudiadas en el contexto de Cataluña. En dicho gráfico se puede comprobar como su localización se corresponde especialmente con la el área metropolitana de Barcelona así como con los espacios turísticos litorales y algunos de los principales destinos turísticos de interior. Parece pues que las respuestas tienen que sobretodo ver con un patrón urbano y que, probablemente, quieren dar respuesta a la demanda de las grandes y medianas ciudades catalanas. Por lo que respecta a la localización de los destinos con los que trabajan estas empresas podemos ver como solo un $22,7 \%$ trabaja con el mismo destino donde se localiza la empresa, lo que tiene que ver con el escaso número de agencias receptivas de la muestra, mientras que parece lógico encontrar un 52,9\% que trabajan con destinos en la región, así como un 40,3\% que trabaja con destinos nacionales y un $69,7 \%$ con destinos internacionales. 


\section{Tabla 2}

\section{PRINCIPALES PRODUCTOS COMERCIALIZADOS}

En porcentaje de empresas que han respondido

\begin{tabular}{|l|r|}
\hline Turismo Cultural & 71,4 \\
Turismo de Naturaleza & 61,3 \\
Turismo Urbano & 53,8 \\
Turismo de Deporte y/o Aventura & 53,8 \\
Sol y Playa & 50,4 \\
Turismo gastronómico & 43,7 \\
Ecoturismo & 37,8 \\
Turismo Rural & 36,1 \\
Turismo de Negocios & 36,1 \\
Turismo de nieve & 31,1 \\
Turismo de Salud & 27,7 \\
Turismo para singles & 25,2 \\
Otros & 25,2 \\
\hline
\end{tabular}

Elaboración propia.

\section{Gráfico 1 \\ LOCALIZACIÓN GEOGRÁFICA DE LAS AGENCIAS DE VIAJE}

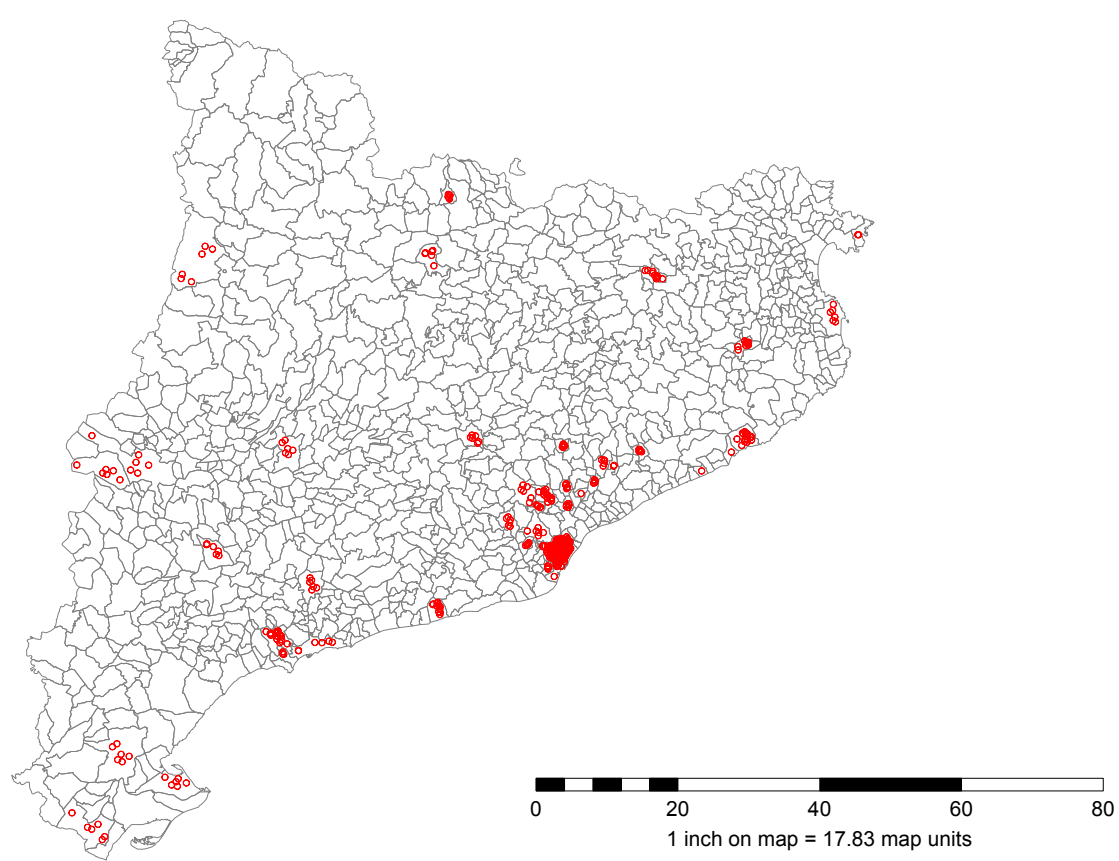

Elaboración propia. 
Por otro lado, un 58,0\% de estos establecimientos son negocios familiares y casi un $80 \%$ de los mismos son microempresas con menos de cinco trabajadores. Casi el mismo porcentaje $(81 \%)$ afirma que la agencia es minorista, predominando aquellas que son emisoras $(48,3 \%)$ o emisoras y receptoras $(30,5 \%)$ y tan solo un $21,2 \%$ son agencias únicamente receptoras. Además, más de un $82 \%$ de las empresas encuestadas son agencias independientes, sin sucursales y un $9,2 \%$ son sucursales de otras agencias que actúan como centrales. Al respecto del perfil de estas agencias, la gran mayoría afirma que es vacacional $(70,6 \%)$, aunque también un porcentaje elevado $(65,5 \%)$ afirma que es especializada y un $31,1 \%$ dice tener también un perfil de negocios. En la tabla 2 podemos ver los porcentajes de respuesta en relación al tipo de producto que comercializan, donde el turismo cultural es el más ofrecido, seguido del de naturaleza, el turismo urbano y el de deporte y aventura. Es además interesante ver como el sol y playa es la quinta opción más ofrecida.

También se observa como un altísimo porcentaje de estas empresas $(82,4 \%)$ tiene presencia en las redes sociales, mayoritariamente en Facebook y Twitter y en algunos casos añadiendo Instagram, Youtube e incluso la red profesional Linkedin. Preguntados los encuestados acerca de por qué los clientes eligen su agencia, la mayor parte estaba de acuerdo o muy de acuerdo con que era por su calidad y también por la proximidad a los clientes, mientras la diferenciación de productos y especialmente el precio ocupaban un porcentaje menor de respuestas. Considerando la salud financiera de los establecimientos, tan solo un $10,2 \%$ de los encuestados afirmaban que era mala, un 45,9\% regular y el resto, cerca de un $44 \%$, afirmaba que era buena $(38,8 \%)$ o muy buena $(5,1 \%)$. Finalmente, preguntados sobre su evolución al respecto en los dos últimos años, un $6 \%$ dice que muy mala, un $12 \%$ mala, un $44 \%$ regular, un $33 \%$ buena y un $5 \%$ muy buena, cifras también positivas en conjunto.

\subsection{Prácticas, razones, barreras y aprendizaje entorno a la sostenibilidad}

Pasando ya al comentario de las variables relacionadas con la sostenibilidad, podemos empezar observando la tabla 3 y los principales resultados en cuanto a las prácticas realizadas.

En este sentido, empezando por las variables medioambientales, podemos ver cómo estas empresas todavía tienen porcentajes relativamente bajos de introducción de la mayor parte de éstas, destacando la información al turista sobre prácticas turísticas medioambientalmente sostenibles o la promoción de las iniciativas de protección medioambiental entre los clientes. Los ecosavings o prácticas ambientales que suponen ahorros de costes (reciclaje, ahorro energético, etc.) tienen, como podría parecer lógico, un menor seguimiento que en otros sectores como el del alojamiento. En el caso de las prácticas sociales y económicas encontramos un mayor seguimiento en algunas variables, que se aproximan al 50\%, destacando la comercialización de productos comprometidos con la realidad del destino y sus comunidades. Entre las prácticas económicas se podrían destacar algunas como la promoción del consumo de productos locales, pero hay que destacar el buen resultado en prácticas como las relacionadas con los sueldos o la contratación de proveedores que favorecen el desarrollo local. Preguntados al respecto de si los clientes de estas empresas valoran la introducción de estas prácticas, un amplio porcentaje de los encuestados $(63,8 \%)$ afirma que en alguna ocasión lo hacen y un 14,3\% dicen que todos los clientes las valoran positivamente. Principalmente la información a este respecto la 
Tabla 3

PRÀCTICAS DE SOSTENIBILIDAD

Número de establecimientos que incorporan las prácticas y porcentaje sobre la muestra

\begin{tabular}{|c|c|c|}
\hline Prácticas medioambientales & $\mathrm{n}$ & $\%$ \\
\hline Informa al turista sobre prácticas turísticas medioambientalmente sostenibles & 38 & 31,9 \\
\hline Promueve entre sus clientes el contribuir a iniciativas de protección medioambiental & 31 & 26,1 \\
\hline Evalúa, de alguna forma, el impacto medioambiental de los productos que comercializa la agencia & 30 & 25,2 \\
\hline Comercializa productos que cumplen una serie de criterios medioambientales certificados & 23 & 19,3 \\
\hline Contrata preferentemente proveedores que reciclan los residuos & 22 & 18,5 \\
\hline Promueve entre sus clientes el ahorro de energía y/o agua & 19 & 16,0 \\
\hline Contrata preferentemente proveedores que implantan prácticas de ahorro energético y/o agua & 17 & 14,3 \\
\hline Contrata preferentemente proveedores que disponen de certificados medioambientales & 16 & 13,4 \\
\hline Contrata preferentemente proveedores que implantan y usan energías alternativas & 16 & 13,4 \\
\hline Promueve entre sus clientes el consumo de productos ecológicos & 16 & 13,4 \\
\hline \multicolumn{3}{|l|}{ Prácticas sociales } \\
\hline Comercializa productos que dan a conocer la realidad de los destinos & 63 & 52,9 \\
\hline Promueve entre sus clientes el respeto por las comunidades de los destinos que se visitan & 56 & 47,1 \\
\hline Apoya el desarrollo local y la conservación del patrimonio & 53 & 44,5 \\
\hline Fomenta las actitudes cívicas entre la clientela & 53 & 44,5 \\
\hline Fomenta activamente el respeto por la lengua propia del territorio & 51 & 42,9 \\
\hline En sus actividades promueve la igualdad de género & 45 & 37,8 \\
\hline Colabora en proyectos sociales & 44 & 37,0 \\
\hline Informa al turista sobre prácticas turísticas socialmente sostenibles & 44 & 37,0 \\
\hline Implanta prácticas de conciliación entre el trabajo y la vida familiar de sus trabajadores & 35 & 29,4 \\
\hline Escoge proveedores que demuestran su sostenibilidad & 35 & 29,4 \\
\hline Coopera con los gestores locales de los destinos donde trabaja & 35 & 29,4 \\
\hline Comercializa productos accesibles que incluyan instalaciones adaptadas para personas discapacitadas & 33 & 27,7 \\
\hline Promueve entre sus clientes el contribuir a iniciativas sociales & 25 & 21,0 \\
\hline Evalúa, de alguna forma, el impacto social de los productos que comercializa la agencia & 23 & 19,3 \\
\hline Comercializa productos que fomentan la solidaridad a través del intercambio y la cultura & 23 & 19,3 \\
\hline Comercializa productos que facilitan la cooperación y el voluntariado en el destino & 19 & 16,0 \\
\hline Proporciona empleo a personas discapacitadas & 6 & 5,0 \\
\hline \multicolumn{3}{|l|}{ Prácticas económicas } \\
\hline Promueve entre sus clientes el consumo de productos locales & 53 & 44,5 \\
\hline El sueldo de sus trabajadores no es inferior a la media del sector & 51 & 42,9 \\
\hline Escoge proveedores que favorecen el desarrollo local & 51 & 42,9 \\
\hline Comercializa productos que buscan beneficiar a las comunidades locales de los destinos visitados & 41 & 34,5 \\
\hline Informa al turista sobre prácticas turísticas económicamente sostenibles & 38 & 31,9 \\
\hline Preferentemente contrata a personas que viven en la localidad donde se "ubica el" establecimiento & 37 & 31,1 \\
\hline Fomenta la progresión de la carrera profesional dentro de la "empresa & 31 & 26,1 \\
\hline Colabora con empresas u organizaciones potencialmente competidoras & 29 & 24,4 \\
\hline Colabora financieramente en proyectos de cooperación & 26 & 21,8 \\
\hline Evalúa, de alguna forma, el impacto económico en la localidad donde se ubica el establecimiento & 25 & 21,0 \\
\hline Promueve entre sus clientes el contribuir a iniciativas solidarias & 19 & 16,0 \\
\hline
\end{tabular}

Fuente: elaboración propia. 
recogen de manera informal (41,3\%), mientras que un 38,5\% afirma que desconoce si los clientes valoran o no la implementación de estas prácticas. La principal vía para comunicar esta implementación a los clientes es presencial, generalmente antes del viaje $(29,9 \%$ de los casos), seguida de la información en la página web $(19,6 \%)$.

En cuanto a las principales razones por las que los propietarios y/o directivos introducen la sostenibilidad en estas empresas, en la Tabla 4 podemos ver como son preponderantes dos razones de carácter altruista, como el compromiso social y la protección del medio ambiente, seguidas por una motivación económica, relacionada con la imagen del negocio, una razón ligada a la mejora del destino y finalmente por una razón personal, relacionada con el estilo de vida.

Tabla 4

MOTIVOS PARA INTRODUCIR LAS PRÁCTICAS DE SOSTENIBILIDAD

Porcentaje de respuestas en la escala Likert. Razones ordenadas a partir de la media de puntos obtenida en la escala de Likert (1 a 5)

\begin{tabular}{|c|c|c|c|c|c|}
\hline & 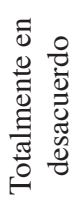 & 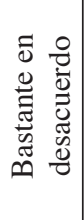 & 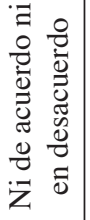 & 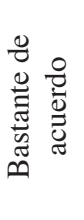 & 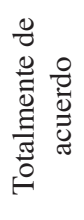 \\
\hline Por mi compromiso con la sociedad & ,0 & 1,9 & 17,3 & 48,1 & 32,7 \\
\hline Para proteger el medio ambiente & ,9 & 3,8 & 18,9 & 38,7 & 37,7 \\
\hline Para que beneficia la imagen de la empresa & 1,0 & 2,0 & 23,5 & 46,9 & 26,5 \\
\hline Para que redunda positivamente en el destino & 1,0 & 2,1 & 28,1 & 37,5 & 31,3 \\
\hline Por cuestiones personales / es mi estilo de vida & 0 & 1,9 & 29,1 & 42,7 & 26,2 \\
\hline Para fidelizar a mis clientes & 2,0 & 7,1 & 25,5 & 39,8 & 25,5 \\
\hline Para ganar nuevos clientes & 5,1 & 4,0 & 31,3 & 39,4 & 20,2 \\
\hline Para mejorar la satisfacción de mis trabajadores & 6,0 & 8,0 & 29,0 & 34,0 & 23,0 \\
\hline Para obtener información útil para gestionar la empresa & 6,5 & 6,5 & 38,7 & 33,3 & 15,1 \\
\hline $\begin{array}{l}\text { Para obtener ventajas competitivas más allá de la reducir } \\
\text { costes }\end{array}$ & 5,4 & 8,6 & 43,0 & 29,0 & 14,0 \\
\hline Para que las tecnologías me facilitan su implementación & 7,3 & 7,3 & 42,7 & 30,2 & 12,5 \\
\hline Para ser pioneros ante futuras normativas relacionadas & 9,3 & 8,2 & 42,3 & 25,8 & 14,4 \\
\hline Para reducir costes & 12,2 & 9,2 & 35,7 & 28,6 & 14,3 \\
\hline Para mejorar la vigilancia del funcionamiento del negocio & 9,5 & 10,5 & 37,9 & 31,6 & 10,5 \\
\hline Para cumplir con requisitos que marca la administración & 15,6 & 9,4 & 34,4 & 28,1 & 12,5 \\
\hline Para obtener mayores beneficios económicos & 11,3 & 15,5 & 38,1 & 23,7 & 11,3 \\
\hline Porque era fácil de llevar a cabo & 8,4 & 15,8 & 50,5 & 20,0 & 5,3 \\
\hline
\end{tabular}




\begin{tabular}{|l|c|c|c|c|c|}
\hline Porque me permiten acceder a asesoramiento y / o redes & 14,0 & 12,9 & 43,0 & 23,7 & 6,5 \\
\hline Para obtener una acreditación que certifique & 15,8 & 11,6 & 42,1 & 23,2 & 7,4 \\
\hline Para cumplir con los requisitos de un turoperador & 17,4 & 12,0 & 42,4 & 17,4 & 10,9 \\
\hline $\begin{array}{l}\text { Para cumplir con los requisitos de una asociación } \\
\text { empresarial }\end{array}$ & 16,1 & 15,1 & 43,0 & 20,4 & 5,4 \\
\hline Para cumplir con los requisitos de un grupo empresarial & 17,0 & 11,7 & 48,9 & 18,1 & 4,3 \\
\hline Para acceder a subvenciones & 19,1 & 18,1 & 41,5 & 17,0 & 4,3 \\
\hline Para acceder más fácilmente a la financiación & 22,6 & 10,8 & 47,3 & 15,1 & 4,3 \\
\hline
\end{tabular}

Fuente: elaboración propia.

Tras estos motivos figuran un conjunto de razones que tienen que ver con la legitimidad antes los grupos de interés (clientes, trabajadores) o directamente con las mejoras en gestión y económicas, predominando la obtención de ventajas competitivas más allá de la reducción de costes. La utilización de las tecnologías resulta también importante para estos encuestados y parece más marginal el hecho de cumplir con las exigencias o expectativas de otros grupos de interés como turoperadores, asociaciones, grupos, administración o el hecho de obtener subvenciones. Si nos fijáramos en las barreras, es la falta de tiempo y de presupuesto la que más reportan los encuestados. Otras barreras, como la consideración de que no sea rentable o que no lo valoren los clientes están en posiciones secundarias.

\section{Tabla 5}

\section{RESULTADOS DE LA INTRODUCCIÓN DE LA SOSTENIBILIDAD}

Porcentaje de respuestas en la escala Likert. Razones ordenadas a partir de la suma de "Bastante de acuerdo" y "Totalmente de acuerdo".

\begin{tabular}{|c|c|c|c|c|c|}
\hline & 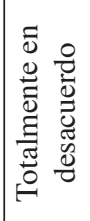 & 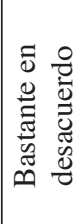 & 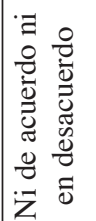 & 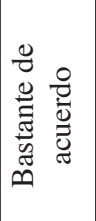 & 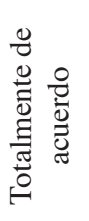 \\
\hline Tenemos un mayor valor de mercado & 1,2 & 5,8 & 46,5 & 32,6 & 14,0 \\
\hline Cumplimos mejor con las expectativas de los consumidores & 2,3 & 2,3 & 48,8 & 34,9 & 11,6 \\
\hline Somos más novedosos en el sector & 0 & 7,0 & 47,7 & 32,6 & 12,8 \\
\hline Competimos mejor con las empresas líderes de nuestro sector & 3,6 & 8,3 & 51,2 & 26,2 & 10,7 \\
\hline Cumplimos mejor con nuestras expectativas de ventas & 2,3 & 10,5 & 61,6 & 20,9 & 4,7 \\
\hline Podemos comercializar más rápidamente & 5,9 & 8,2 & 65,9 & 16,5 & 3,5 \\
\hline Reducimos nuestros costes de gestión & 5,8 & 14,0 & 60,5 & 15,1 & 4,7 \\
\hline Obtenemos más beneficios & 5,9 & 14,1 & 65,9 & 8,2 & 5,9 \\
\hline
\end{tabular}

Fuente: elaboración propia. 
Si consideramos los resultados de la introducción de estas prácticas, los encuestados valoran de manera muy positiva las mejoras derivadas, como se puede apreciar en la Tabla 5, donde destaca la posibilidad de tener un mayor valor de mercado, o cumplir mejor con las expectativas de los consumidores. Además, como también se puede ver, el hecho de reducir costes u obtener más beneficios directamente no tiene la misma consideración y resulta menos importante para los encuestados. Finalmente, como se puede observar en la tabla 6, indagados acerca de sus expectativas sobre la sostenibilidad en el sector, cerca de un $60 \%$ de los encuestados piensa que el número de agencias que comercializará productos sostenibles aumentará en el futuro, que las TIC jugarán un papel protagonista en este escenario y que las perspectivas de negocio para su agencia serán buenas. En general, los porcentajes de desacuerdo con estas preguntas no llegan al $15 \%$ en ningún caso.

\section{Tabla 6}

\section{PERSPECTIVAS DE FUTURO A PROPÓSITO DE LA SOSTENIBILIDAD}

Porcentaje de respuestas en la escala Likert. Razones ordenadas a partir de la suma de "Bastante de acuerdo" y "Totalmente de acuerdo".

\begin{tabular}{|c|c|c|c|c|c|}
\hline & 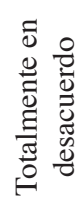 & 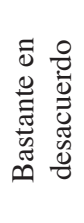 & 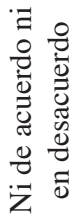 & 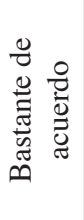 & 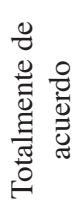 \\
\hline $\begin{array}{l}\text { En los próximos años las perspectivas de negocio para mi } \\
\text { agencia serán buenas }\end{array}$ & 2,0 & 7,0 & 31,0 & 46,0 & 14,0 \\
\hline $\begin{array}{l}\text { El número de "agencias que comercialicen productos } \\
\text { relacionados con el turismo responsable aumentará } \\
\text { notablemente }\end{array}$ & 2,0 & 11,8 & 26,5 & 42,2 & 17,6 \\
\hline $\begin{array}{l}\text { Las TIC serán un factor que impulsarán estrategias de } \\
\text { transparencia en los procesos de turismo responsable }\end{array}$ & 1,0 & 5,1 & 33,3 & 40,4 & 20,2 \\
\hline
\end{tabular}

Fuente: elaboración propia.

\subsection{Comportamiento sostenible y caracterización del negocio}

$\mathrm{Al}$ respecto de las relaciones entre las variables que definen el comportamiento sostenible de estas empresas y las características genéricas de estas empresas, y comenzando por los motivos para introducir la sostenibilidad, se observó la posible existencia de factores que pudieran agrupar estas razones o motivos, obteniéndose los tres factores que se pueden ver en la tabla 7. El primer factor, al que hemos llamado "Gestión" agrupa una serie de motivos distintivos de los intereses económicos y/o relacionados con la gestión de la agencia así como con la relación con los grupos de interés relacionados directamente con su funcionamiento. El segundo factor, al que hemos llamado "Altruismo" incluye a los motivos relacionados con el compromiso social, ambiental o con la protección del destino, 
además del motivo relacionado con el estilo de vida, razones que generalmente la literatura también ha considerado de manera agregada. En tercer lugar, encontramos un tercer factor, al que hemos llamado "Clientes", y que agrupa a los motivos relacionados con la imagen de la agencia y en general la orientación y respuesta al cliente, a los que se añade el elemento tecnológico, generalmente muy relacionado con este aspecto.

Tabla 7

\section{ANÁLISIS FACTORIAL DE LAS VARIABLES RELACIONADAS CON LOS MOTIVOS PARA INTRODUCIR LA SOSTENIBILIDAD}

Matriz de componentes rotados.

\begin{tabular}{|c|c|c|c|}
\hline & Gestión & Altruismo & Clientes \\
\hline $\begin{array}{l}\text { Para cumplir con los requisitos indicados por una asociación } \\
\text { empresarial }\end{array}$ &, 883 &,- 016 & ,126 \\
\hline $\begin{array}{l}\text { Para cumplir con los requisitos indicados por el grupo al que } \\
\text { pertenezco }\end{array}$ &, 834 &,- 077 &, 061 \\
\hline Para reducir costes &, 831 &,- 006 & ,196 \\
\hline Para cumplir con los requisitos indicados por un turoperador &, 818 &,- 009 & ,136 \\
\hline Para obtener mayores beneficios económicos & ,798 & ,138 &, 151 \\
\hline $\begin{array}{l}\text { Para mejorar la vigilancia / control del funcionamiento del } \\
\text { negocio }\end{array}$ & ,784 & ,088 & ,323 \\
\hline Para que puedo acceder a asesoramiento y / o redes & ,769 &,- 004 &, 325 \\
\hline Para obtener una certificación que lo acredite & ,768 &, 016 & ,306 \\
\hline Para cumplir con requisitos legales que marca la administración & ,765 &,- 060 &, 127 \\
\hline Para ser pioneros ante futuras normativas relacionadas & ,759 & ,143 & ,283 \\
\hline Para acceder más fácilmente a la financiación & ,727 &,- 213 & ,319 \\
\hline $\begin{array}{l}\text { Para obtener ventajas competitivas más allá de la reducción de } \\
\text { costes }\end{array}$ & ,704 & ,366 & ,064 \\
\hline Para acceder a subvenciones & ,686 &,- 200 & ,419 \\
\hline Para que obtengo información útil para la gestión de la empresa & ,672 &, 190 & ,445 \\
\hline Para mejorar la satisfacción de mis trabajadores &, 578 & ,290 & ,461 \\
\hline Porque era fácil de llevar a cabo &, 552 & ,009 &, 430 \\
\hline Por cuestiones personales / es mi estilo de vida & ,035 & ,846 &,- 075 \\
\hline Por mi compromiso con la sociedad &,- 084 & ,837 & ,235 \\
\hline Para proteger el medio ambiente &, 085 & ,781 & ,270 \\
\hline Para que redunda positivamente en el destino &,- 082 &, 758 &, 100 \\
\hline Para que beneficia la imagen de la empresa & ,176 & ,342 & ,795 \\
\hline $\begin{array}{l}\text { Para que las tecnologías me facilitan su implementación y } \\
\text { difusión }\end{array}$ & ,245 & ,135 & ,745 \\
\hline Para ganar nuevos clientes &, 529 & ,093 &, 567 \\
\hline Para fidelizar a mis clientes & ,435 & ,443 &, 531 \\
\hline
\end{tabular}

Método de extracción: Análisis de componentes principales. Método de rotación: Normalización Varimax con Kaiser. La rotación ha convergido en 5 iteraciones. Fuente: elaboración propia. 
Parece pues que existen tres factores que pueden explicar el amplio conjunto de motivaciones para ser sostenible de estas compañías y que por tanto muestran diferentes comportamientos o tendencias predominantes. Por otro lado, nos interesaba también saber si existía algún tipo de relación entre estos factores con otras variables y para ello llevamos a cabo un análisis de correlación (Pearson) en el que surgieron diferentes relaciones significativas. En cuanto al factor relacionado con la gestión del negocio, se observó una relación positiva con los perfiles de agencias dedicadas al turismo de negocios y que ofrecen productos relacionados con este segmento. De la misma manera se obtuvo una relación negativa con el hecho de ofrecer productos ecoturísticos, lo que además ayuda a perfilar bastante bien a este factor. También resulta oportuno ver la fuerte relación con el hecho de trabajar con destinos a nivel nacional. Es además el único factor que tiene alguna relación significativa con los resultados que declaran los propietarios o gestores, en este caso claramente negativa.

Si por otro lado observamos las posibles relaciones entre el factor "Altruismo" y las variables de caracterización de estas empresas podemos empezar señalando la relación positiva con el hecho que la propietaria o gestora sea mujer, y que tenga una mayor cualificación. La siguiente relación significativa la encontramos en el perfil de la agencia, en este caso muy adverso al vacacional y muy propenso a presentarse como agencia especializada. Algo similar se detecta en cuanto a los productos, donde se observa una relación negativa con productos tradicionales como el sol y playa o el turismo de nieve y positiva en relación a la oferta de ecoturismo. Otra relación se encuentra con el hecho de trabajar con destinos prioritariamente locales y también, de manera muy especial, de trabajar con redes sociales. Además, este factor se relaciona de manera especialmente negativa con el hecho de afirmar que se dispone de una estrategia formal de sostenibilidad. Finalmente, en el caso del factor "Clientes" existen menos relaciones significativas, pero también ilustrativas de este perfil, como el hecho de destacar el género masculino entre los propietarios, con la antigüedad del negocio, con el hecho de no ser agencias familiares, así como también con ofrecer productos relacionados con el turismo de negocios.

Al respecto de las barreras también se encontraron tres factores. Un primer factor al que hemos llamado "Falta de Recursos" se relaciona con la falta de recursos (falta de tiempo, de presupuesto, la competencia no lo hace, falta de formación, el cliente no lo pide, la administración no lo ha impulsado, no hay suficientes fuentes de información, no hay tecnologías que lo faciliten). Un segundo factor denominado "Grupos de Interés" se relaciona con la falta de impulso por parte de los grupos de interés (la asociación a la que pertenece no lo cree conveniente, las experiencias que conoce no son convincentes, no contribuye a mejorar la imagen de la agencia o no ve el interés). Finalmente, un tercer factor nombrado "Desconfianza" agruparía las barreras que tienen que ver con la falta de confianza (no cree que sea lo suficientemente rentable, aunque tengo formación lo veo demasiado complicado o si lo hago nadie lo valorará). En cuanto a las correlaciones, el factor "Falta de Recursos" se relaciona positivamente con la oferta del producto gastronómico y negativamente con la satisfacción con los resultados, mientras que el factor "Desconfianza" lo hace negativamente con el trabajar con destinos regionales. Por su parte el factor "Grupos de Interés" se relaciona negativamente con el nivel de estudios del propietario, con el hecho de que la empresa sea familiar, positivamente con ofrecer productos de nieve y negativamente con los de naturaleza, ecoturismo, cultura y gastronomía. Este mismo factor se relaciona positivamente con el hecho de 
trabajar con destinos nacionales y con que los clientes elijan la agencia por precio. Además, también existe una correlación negativa con la presencia en las redes sociales.

Por lo que hace a la relación entre otras variables, las prácticas ambientales se correlacionan positivamente con el género femenino y con el hecho de que la empresa sea familiar mientras el hecho que la agencia tenga un perfil especializado se correlaciona positivamente con la introducción de prácticas ambientales y sociales. Por otro lado, ofrecer el producto de sol y playa se correlaciona negativamente con la introducción de prácticas de los tres tipos, mientras ofrecer productos de turismo rural y de naturaleza, así como ecoturismo se relaciona positivamente con la introducción de los tres tipos de prácticas. Además, ofrecer turismo cultural se correlaciona positivamente con la introducción de prácticas de tipo social. Por otro lado, trabajar con destinos nacionales se correlaciona negativamente con la introducción de prácticas de sostenibilidad y positivamente con el hecho que los clientes elijan a la agencia por su calidad y por su diferenciación. La introducción de prácticas se correlaciona también negativamente con el hecho de disponer de una estrategia formal de sostenibilidad y también una correlación significativa con algunos de los motivos para introducirlas, especialmente para el caso de la protección medioambiental, el compromiso social, el estilo de vida o la protección del destino. Esto se manifiesta de manera clara al observar la correlación entre las prácticas y los factores que agrupaban estos motivos, donde el factor "Altruismo" es el único que se correlaciona con las prácticas y lo hace de manera positiva para los ejes ambiental, social y económico, como se observa en la tabla 8.

\section{Tabla 8}

\section{RELACIÓN ENTRE LA INTRODUCCIÓN DE PRÁCTICAS DE SOSTENIBILIDAD Y LOS MOTIVOS PARA INTRODUCIRLAS}

Regresiones lineales. Valores $\beta$ s, símbolos de significación, Regresiones F-ANOVAs y R².

\begin{tabular}{|c|c|c|c|c|c|c|}
\hline & & \multicolumn{2}{|c|}{$\begin{array}{l}\text { Coeficientes no } \\
\text { estandarizados }\end{array}$} & \multirow{2}{*}{$\begin{array}{c}\begin{array}{c}\text { Coeficientes } \\
\text { tipificados }\end{array} \\
\text { Beta }\end{array}$} & \multirow{2}{*}{$\mathbf{t}$} & \multirow{2}{*}{ Sig. } \\
\hline & & B & $\begin{array}{l}\text { Error } \\
\text { típ. }\end{array}$ & & & \\
\hline \multirow{4}{*}{$\begin{array}{c}\text { Dependiente: Prácticas } \\
\text { ambientales }\end{array}$} & Constante & ,199 & 023 & & 8,787 & 000 \\
\hline & Gestión &,- 009 & 023 &,- 036 &,- 384 & ,702 \\
\hline & Altruismo & ,142 & 023 & ,588 & 6,238 & ,000 \\
\hline & Clientes &,- 015 & 023 &,- 062 &,- 655 &, 514 \\
\hline \multirow{4}{*}{ Dependiente: Prácticas sociales } & Constante & ,341 & 026 & & 13,189 & ,000 \\
\hline & Gestión &,- 014 & 026 &,- 052 &,- 525 & 601 \\
\hline & Altruismo & ,139 & 026 &, 529 & 5,340 &, 000 \\
\hline & Clientes & 009 & 026 &, 032 & ,328 & ,744 \\
\hline \multirow{4}{*}{$\begin{array}{c}\text { Dependiente: Prácticas } \\
\text { económicas }\end{array}$} & Constante & ,327 & 024 & & 13,422 & ,000 \\
\hline & Gestión &,- 026 & 025 &,- 109 & $-1,073$ & ,287 \\
\hline & Altruismo & ,118 & 025 & ,488 & 4,818 &, 000 \\
\hline & Clientes &,- 004 &, 025 &,- 014 &,- 143 & 887 \\
\hline
\end{tabular}

Fuente elaboración propia. Nota: $* * *=\mathrm{p}<0.001$. Sin problemas de multicolinealidad con IVF o índices de condición. 
Si realizamos el mismo ejercicio en relación a las barreras para introducir la sostenibilidad y las propias prácticas (tabla 9) observamos como la introducción de prácticas ambientales se relaciona negativamente con el factor "Falta de Recursos" así como con el "Grupos de Interés". Por su parte, la introducción de prácticas sociales se relaciona significativamente y de manera negativa con las barreras que tienen que ver con este factor "Grupos de Interés" y la introducción de prácticas económicas lo hace con éstas y con el factor que agrupa a las barreras relacionadas con la desconfianza en la utilidad de implementar estas prácticas.

\section{Tabla 9 \\ RELACIÓN ENTRE LA INTRODUCCIÓN DE PRÁCTICAS DE SOSTENIBILIDAD Y LAS BARRERAS PARA INTRODUCIRLAS}

Regresiones lineales. Valores $\beta$ s, símbolos de significación, Regresiones F-ANOVAs y R².

\begin{tabular}{|c|c|c|c|c|c|c|}
\hline & \multicolumn{2}{|c|}{$\begin{array}{l}\text { Coeficientes no } \\
\text { estandarizados }\end{array}$} & \multirow{3}{*}{$\begin{array}{c}\begin{array}{c}\text { Coeficientes } \\
\text { tipificados }\end{array} \\
\text { Beta }\end{array}$} & \multirow{3}{*}{$\begin{array}{c}\mathbf{t} \\
8,692\end{array}$} & \multirow{3}{*}{$\begin{array}{l}\text { Sig. } \\
, 000\end{array}$} \\
\hline & & B & $\begin{array}{l}\text { Error } \\
\text { típ. }\end{array}$ & & & \\
\hline \multirow{4}{*}{$\begin{array}{c}\text { Dependiente: Prácticas } \\
\text { ambientales } \\
\text { (F ANOVA }=4,611(* * *) \\
\left.\text { y R }^{2}=0,388\right)\end{array}$} & (Constante) & ,212 & ,024 & & & \\
\hline & $\begin{array}{l}\text { Falta de } \\
\text { Recursos }\end{array}$ &,- 049 &, 025 &,- 207 & $-1,980$ &, 051 \\
\hline & Grupos Interés &,- 073 &, 025 &,- 308 & $-2,952$ & ,004 \\
\hline & Desconfianza &,- 027 &, 025 &,- 114 & $-1,095$ & ,277 \\
\hline \multirow{4}{*}{$\begin{array}{c}\text { Dependiente: Prácticas } \\
\text { sociales } \\
\text { (F ANOVA }=6,564(* * *) \\
\left.\text { y R }^{2}=0,449\right)\end{array}$} & (Constante) & ,348 & ,026 & & 13,544 &, 000 \\
\hline & $\begin{array}{l}\text { Falta de } \\
\text { Recursos }\end{array}$ &,- 008 &, 026 &,- 033 &,- 323 & ,747 \\
\hline & Grupos Interés &,- 095 & ,026 &,- 372 & $-3,682$ & ,000 \\
\hline & Desconfianza &,- 063 & 026 &,- 249 & $-2,456$ &, 016 \\
\hline \multirow{4}{*}{$\begin{array}{c}\text { Dependiente: Prácticas } \\
\text { económicas } \\
\text { (F ANOVA }=4,344(* * *) \\
\left.\text { y R }^{2}=0,378\right)\end{array}$} & (Constante) & ,336 & ,025 & & 13,321 &, 000 \\
\hline & $\begin{array}{l}\text { Falta de } \\
\text { Recursos }\end{array}$ &,- 026 &, 025 &,- 108 & $-1,027$ & ,308 \\
\hline & Grupos Interés &,- 059 &, 025 &,- 244 & $-2,327$ &, 023 \\
\hline & Desconfianza &,- 065 &, 025 &,- 268 & $-2,556$ &, 013 \\
\hline
\end{tabular}

Fuente elaboración propia. Nota: $* * *=\mathrm{p}<0.001$. Sin problemas de multicolinealidad con IVF o índices de condición.

Para entender mejor la dimensión de innovación que acompaña la introducción de medidas de sostenibilidad se ha confeccionado un análisis factorial. Se desprende la existencia de dos factores, un primer factor que etiquetamos como "Mejoras internas" y que agrupa mejoras que se producen especialmente en el funcionamiento de la empresa y en la obtención de mayores beneficios (comercialización más rápida, más beneficios, cumple sus expectativas de ventas, reduce los costes de gestión o considera que puede competir mejor con las empresas líderes del sector). Por otro lado, un segundo factor que etiquetamos como "Mejoras de mercado" agrupa a las mejoras que se relacionan directamente con el mercado (es más novedosa para el mercado, tiene un mayor valor de mercado y cumple mejor con las expectativas de los consumidores). 
Finalmente, al respecto de la relación entre las razones y barreras para introducir la sostenibilidad con estas mejoras en el negocio, en la tabla 10 se puede apreciar como las mejoras de tipo interno dependen de manera positiva de aquellas razones o motivos vinculados a la gestión de la empresa y también a la orientación al cliente, así como también de las barreras relacionadas con el reporting a los grupos de interés. Por su parte las mejoras de mercado derivadas de la introducción de prácticas sostenibles dependen positivamente de que los propietarios las introduzcan por motivos de tipo altruista o en relación con su estilo de vida y negativamente de que éstos tengan como barrera para introducirlas la desconfianza.

Tabla 10

\section{RELACIÓN ENTRE LOS RESULTADOS DE IMPLEMENTAR PRÁCTICAS DE SOSTENIBILIDAD Y LOS MOTIVOS Y BARRERAS PARA INTRODUCIRLA}

Regresiones lineales. Valores b s, símbolos de significación, Regresiones F-ANOVAs y R².

\begin{tabular}{|c|c|c|c|c|c|c|}
\hline \multirow{2}{*}{\multicolumn{2}{|c|}{ B }} & \multicolumn{2}{|c|}{$\begin{array}{l}\text { Coeficientes no } \\
\text { estandarizados }\end{array}$} & \multirow[t]{2}{*}{$\begin{array}{l}\text { Coeficientes } \\
\text { tipificados }\end{array}$} & \multirow{2}{*}{$\mathbf{t}$} & \multirow{2}{*}{ Sig. } \\
\hline & & $\begin{array}{l}\text { Error } \\
\text { típ. }\end{array}$ & Beta & & & \\
\hline \multirow{7}{*}{$\begin{array}{c}\text { Dependiente: Mejoras } \\
\text { internas }\end{array}$} & (Constante) &,- 004 &, 105 & &,- 038 & ,970 \\
\hline & Motivos: Gestión &, 380 &, 124 & ,361 & 3,076 &, 003 \\
\hline & $\begin{array}{l}\text { Motivos: } \\
\text { Altruismo }\end{array}$ & ,206 &, 127 & ,206 & 1,620 &, 111 \\
\hline & Motivos: Clientes &, 262 &, 114 &, 243 & 2,300 &, 025 \\
\hline & $\begin{array}{c}\text { Barreras: Falta de } \\
\text { Recursos }\end{array}$ &,- 125 & ,129 &,- 113 &,- 966 &, 339 \\
\hline & $\begin{array}{c}\text { Barreras: Grupos } \\
\text { Interés }\end{array}$ &, 532 &, 142 & ,490 & 3,746 & 000 \\
\hline & $\begin{array}{c}\text { Barreras: } \\
\text { Desconfianza }\end{array}$ &,- 170 &, 120 &,- 144 & $-1,411$ & ,164 \\
\hline \multirow{7}{*}{ 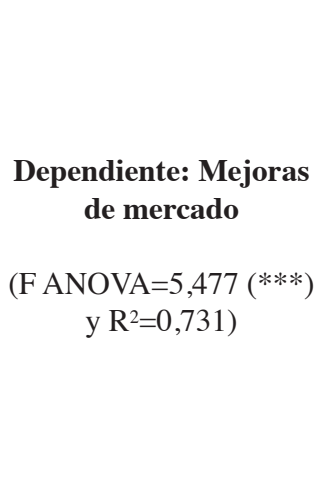 } & (Constante) &,- 114 &, 112 & & $-1,014$ &, 315 \\
\hline & Motivos: Gestión & ,227 & ,133 & ,226 & 1,713 & ,093 \\
\hline & $\begin{array}{l}\text { Motivos: } \\
\text { Altruismo }\end{array}$ & ,306 & ,136 &, 320 & 2,247 & ,029 \\
\hline & Motivos: Clientes &, 079 &, 122 &, 077 & ,646 &, 521 \\
\hline & $\begin{array}{c}\text { Barreras: Falta de } \\
\text { Recursos }\end{array}$ &,- 176 &, 139 &,- 166 & $-1,265$ &, 211 \\
\hline & $\begin{array}{c}\text { Barreras: Grupos } \\
\text { Interés }\end{array}$ &,- 242 &, 152 &,- 233 & $-1,586$ &, 119 \\
\hline & $\begin{array}{c}\text { Barreras: } \\
\text { Desconfianza }\end{array}$ &,- 301 &, 129 &,- 268 & $-2,331$ & ,024 \\
\hline
\end{tabular}

Fuente elaboración propia. Nota: $* * *=p<0.001$. Sin problemas de multicolinealidad con IVF o índices de condición. 


\section{DISCUSIÓN}

Los resultados recopilados en el artículo analizan por primera vez de manera amplia el alcance de la introducción de prácticas de sostenibilidad en el subsector de la intermediación, con especial atención al contexto de las pequeñas y medianas agencias de viaje y permitiendo observar el grado de divergencia existente entre diferentes perfiles de empresas dentro del mismo. Como se comentó en el marco teórico, la literatura ha identificado estos perfiles de empresas para la gran empresa turística (Lee y Park, 2009; Molina, Claver et al., 2009; Inoue y Lee, 2011) así como para las pymes del subsector del alojamiento turístico (Tzschentke, Kirk et al., 2008; Sheldon y Park, 2010; Barton, Schlenker et al., 2012; Sampaio, Thomas et al., 2012) estableciendo tres grandes grupos de motivaciones que influyen tanto en la implementación de la sostenibilidad como en algunos casos en otras variables de negocio, incluyendo los resultados (Garay y Font, 2012).

En este sentido, en el presente estudio se ha podido constatar cómo, de la misma forma que sucedía en otros subsectores, la visión de los propietarios y/o gestores de las agencias sobre la sostenibilidad dista de ser homogénea, detectándose factores que explican las diferentes visiones y comportamientos. En primer lugar hay que señalar que los factores corporativos vinculados con los procesos de gestión empresarial o la búsqueda de mercados (Salzmann, Ionescu-Somers et al., 2005; Carroll y Shabana, 2010) están también presentes al explicar el posicionamiento de las agencias en términos de sostenibilidad, pero son otro tipo de factores como el altruismo y el estilo de vida (Sampaio, Thomas et al., 2012), los que explican más claramente las diferencias contrastadas. La interpretación sobre la presencia de altruismo no tiene tanto que ver con la madurez de las empresas en su aproximación a la sostenibilidad como con un factor personal aplicado a un determinado modelo de negocio de intermediación. El altruismo como factor explicativo se relaciona con un perfil determinado de agencia de viajes (pyme y especializada) pero también con perfil de propietario (el género femenino o el nivel de cualificación elevado son factores discriminantes) y lo que resulta bastante convincente, con el uso de las tecnologías de la información y de las redes sociales. La apuesta personal se demuestra por ejemplo por el hecho de que en un $63 \%$ de los casos las prácticas de sostenibilidad se implementan sin tener en cuenta las exigencias de los clientes. Este resultado puede interpretarse como un elemento de confianza en esta apuesta personal por un proyecto innovador que puede ser de interés para el mercado y, sobre todo, que satisface personalmente al emprendedor.

La motivación hacia la incorporación de la sostenibilidad en este modelo de negocio representado por pequeñas y medianas pymes minoristas especializadas se asocia, por lo tanto, especialmente a un factor personal. Desde este punto de vista, la motivación hacia la introducción de prácticas de sostenibilidad parece más ligada a un proyecto empresarial vital que no a la búsqueda de nuevos mercados o de resultados económicos a corto plazo. En este sentido una futura línea de investigación, ya iniciada en otros ámbitos del turismo (Sampaio, Thomas et al., 2012), deberá profundizar acerca de si la valoración de la experiencia personal y el sistema de creencias y modo de vida de partida son factores que influyen al explicar las diferencias sobre la motivación de los propietarios de agencias. Por otro lado, los factores que se asocian a las barreras a la introducción de prácticas de 
sostenibilidad y a diferencia de lo reportado en los grandes touroperadores (Baddeley, Font et al., 2011) tienen que ver con la desconfianza y la percepción de que faltan mercados potenciales que demanden la sostenibilidad. Algunas de estas empresas perciben que la realidad del sector les impide jugar con confianza la carta de la sostenibilidad en el terreno de la comercialización y apertura de nuevos mercados, pero también las barreras se relacionan con la falta de recursos y con la falta de interés corporativa. Este planteamiento de reticencias a la introducción de prácticas de sostenibilidad se explica por un perfil de agencias conformado por pymes más reactivas que proactivas y con escasa capacidad de tiempo para formación e innovación de producto o con escaso empoderamiento de sus trabajadores en la toma de decisiones que permita reforzar el factor personal.

Finalmente, el análisis de los resultados de la introducción de prácticas de sostenibilidad permite ofrecer una visión de las expectativas que las agencias tienen sobre este ámbito. Los resultados tienen que ver sobre todo con mejoras en la gestión y funcionamiento de la agencia, así como con la obtención de resultados que permitan alcanzar nuevos mercados. Las expectativas son, hasta cierto punto, opuestas a las de los factores de barreras. Es decir, las agencias ven como barrera la falta de un mercado consolidado, pero, al mismo tiempo, ven como resultado de la introducción de prácticas de sostenibilidad una posible mejora en cuanto a mayor acceso a nuevos mercados. Nuevamente el elemento personal y de emprendeduría explica estos resultados: las agencias que apuestan por la sostenibilidad confían en el carácter vital e innovador de su proyecto para obtener beneficios, mejorar su competitividad y acceder a los mercados, de tal forma que el business case aparece también aquí, aunque sea implícitamente.

Una segunda aportación tiene que ver con las respuestas de las diferentes tipologías de agencias a la introducción de prácticas de sostenibilidad, de la misma manera que se ha analizado anteriormente para diferentes subsectores turísticos (Brønn y Vidaver-Cohen, 2009; Molina, Claver et al., 2009; Sheldon y Park, 2010; Garay y Font, 2012; Sampaio, Thomas et al., 2012). Este estudio pone de manifiesto la existencia de tres perfiles diferentes de agencias de viaje en relación a su comportamiento con la sostenibilidad. El primero es el de una agencia de viajes que se abre a la sostenibilidad como elemento de mejora de su competitividad a través de la diferenciación de negocio en relación a los competidores. Todos los factores asociados a este perfil guardan relación con la posibilidad de mejorar su posición competitiva. Es un perfil que puede responder a cualquier tipo de agencia, pero sobre todo afecta a agencias convencionales de tamaño medio y que, por lo tanto, tienen una estructura de negocio que les facilita la introducción de prácticas de sostenibilidad como factor de competitividad. Un segundo tipo de agencia tiene un perfil marcadamente oportunista y orientado a ganar cuota de mercado con la sostenibilidad. Estas agencias, también de tamaño medio, aunque de mayores dimensiones que las del grupo anterior, y con elevada capacidad tecnológica, introducen la sostenibilidad como estrategia de mercado y de "lavado de imagen" por lo que su misión, en relación al a sostenibilidad, responde más a la obtención de objetivos finalistas que a una visión determinada del mundo. Finalmente existe un tercer perfil de agencia que encaja perfectamente con la del pequeño emprendedor concienciado. Son pymes altamente especializadas y con un compromiso que admite relaciones de compromiso más allá del refuerzo competitivo de la empresa a partir de relaciones win-win con los destinos, la sociedad y los propios clientes. 
La existencia de estos tres perfiles pone de relieve también tres niveles de implicación con la sostenibilidad, cuyo conocimiento puede resultar de vital importancia para los actores sociales y planificadores del sector de la intermediación. Los emprendedores con elevada motivación personal, alto carácter de innovación y de riesgo empresarial implementan prácticas muy diferentes a los otros dos perfiles, que entienden la sostenibilidad como un factor más relacionado con la supervivencia empresarial o con el aumento de la competitividad. En el primer caso suele tratarse de empresas pioneras e innovadoras que requieren una mayor visibilidad y posicionamiento en los mercados. Su sensibilidad hacia la captación de nuevos mercados no depende tanto de la creación de campañas de publicidad como de la proximidad natural y empatía con la demanda. Por lo tanto, una primera recomendación sería sugerir algún tipo de asociación empresarial de las pymes que pertenecen a este perfil y la confección de un sello de garantía o de calidad empresarial en sostenibilidad. A pesar de ocupar un alto porcentaje de las estructuras de la oferta del subsector, en el contexto global estas empresas asumen un pequeño porcentaje del mercado, pero tienen un marcado efecto demostrativo que permite impulsar los procesos de sostenibilidad al resto de perfiles de agencias.

El segundo perfil responde a agencias "convencionales" con una mayor cuota de mercado, pero con una visión limitada de la sostenibilidad a la obtención de prácticas para el aumento de su competitividad. En este caso se podría considerar una acción corporativa a partir de las asociaciones de agencias de viaje existentes para proporcionar ayuda específica, recomendaciones y recursos a las agencias interesadas en incrementar su compromiso con la sostenibilidad. Por último, existe un tercer perfil de agencias que, a pesar de que probablemente tienen las mayores cuotas de mercado usan solamente las prácticas de sostenibilidad para limpiar su imagen y atraer a algunos segmentos de mercado. En este caso las prácticas deberían pasar por las auditorías para la obtención/exclusión de sellos de calidad en sostenibilidad, pero sobre todo por la formación y por la capacitación del empoderamiento de sus empleados en la toma de decisiones acerca de la sostenibilidad.

\section{CONCLUSIONES}

El presente artículo supone una contribución pionera al análisis del comportamiento sostenible del sector de la intermediación. En primer lugar, se constata que algunos factores de motivación o de barrera a la introducción de prácticas de sostenibilidad son coherentes con lo que apuntan otros trabajos relacionados con el sector turístico. En este sentido el altruismo y el estilo de vida aparecen también entre los principales factores motivadores para implementar un mayor número de prácticas de sostenibilidad y para que éstas presenten unas características más avanzadas y requieran innovaciones organizativas. Por otro lado, entre las barreras son la falta de recursos y la falta de conocimientos las que aparecen como aspectos coincidentes en relación a otros estudios sectoriales. Otra propuesta teórica tiene que ver con los factores de motivación obtenidos según cada perfil de empresa. Por un lado la posición de los agentes tradicionales respecto a los factores de motivación y barreras a la introducción de prácticas de sostenibilidad coincide con la literatura existente (Davies y Downward, 2007; Sigala, 2008; Dodds y Kuehnel, 2010; Baddeley, Font et al., 2011), siendo la adquisición de ventajas competitivas el factor que 
mejor explica la introducción de dichas prácticas, así como la falta de confianza en la captación de nuevos mercados es el factor que mejor explica su ausencia. Sin embargo, los agentes asimilables al perfil de emprendedor que establece una pyme como proyección de un proyecto vital tienen una respuesta sensiblemente diferente. En este caso no se destacan las ventajas competitivas o el ahorro en costes, sino la legitimidad que les ofrece posicionarse positivamente en relación a la sostenibilidad ofrecida.

El estudio nos lleva también a una reflexión al respecto de lo que se podría considerar como producción sostenible en el ámbito de la intermediación. En este sentido, nos parece prudente seguir a Clarke (1997) para no proponer definiciones cerradas sino entender que la producción sostenible en este ámbito debe ser un concepto en permanente evolución y sujeto a revisión a medida que se vayan alcanzado los objetivos iniciales. Este sería sobretodo el caso del perfil de agencias más comprometidas con la sostenibilidad y que relacionan su implementación con mejoras de mercado. Etiquetar a su producción como sostenible sería probablemente incorrecto, pues el objetivo debería ser el seguir minimizando impactos negativos y mejorando los positivos sobre los grupos de interés con los que interactúan. De la misma manera, para los perfiles más convencionales de agencias que todavía no introducen habitualmente ninguna práctica de responsabilidad en sus productos o que si lo hacen se limitan a prácticas básicas, de carácter operativo y con el fin de reducir costes, existe precisamente una gran potencialidad para introducir otro tipo de prácticas de carácter más avanzado y que requieren por lo general transformaciones organizativas. En estos casos más que decir que la producción es insostenible se debería entender que todavía tienen un amplio margen para hacer que ésta sea más sostenible.

Como cualquier estudio de este tipo, el presente tiene una serie de limitaciones. La primera tiene que ver con el hecho de que la muestra se corresponde de manera bastante exacta a la población por lo que respecta a una estructura del sector de la intermediación especialmente sesgada a la pyme. Como se ha comentado anteriormente, la pyme copa la estructura del sector, pero no la cuota de mercado, que sigue en manos de las grandes compañías. Sería por lo tanto muy interesante como línea de investigación futura el poder comparar estos datos con los resultantes de una encuesta en estos perfiles. Por otro lado, desde la perspectiva de las motivaciones y barreras, aunque el estudio contempla las que generalmente se han reportado en el sector turístico, quizás se podría realizar un análisis previo al respecto de posibles motivaciones y barreras específicas del sector. De la misma manera no se valoran los posibles antecedentes de estas motivaciones, ya sean especialmente valores como creencias. En este sentido se podrían utilizar modelos aplicados en otros contextos y que relacionan a los valores con las motivaciones y éstas con las actitudes y las intenciones de actuar de determinada forma (en nuestro caso actuar a través de la implementación de la sostenibilidad).

Otra clara limitación del trabajo se encuentra en el hecho de que se presentan las prácticas de sostenibilidad sin definir previamente a través de qué mecanismos se llevan a cabo y garantizan dichas prácticas. Aunque como se ha comentado, el objetivo del presente estudio es relacionar las motivaciones, barreras y mejoras del negocio con dichas prácticas, conocer las dinámicas que las hace posible y los mecanismos de garantía de su cumplimiento podría aportar una información especialmente interesante para entender cómo se traducen dichas prácticas en mejoras de negocio, ya sean internas como externas. 
En este sentido cabría observar tanto los sistemas de gestión más formalizados que realizan sobretodo empresas de mayor dimensión como la realización de análisis cualitativo que observaran como se introducen las prácticas en las pymes, generalmente en entornos menos formales (o que incluso en ocasiones rehúyen la sistematización al respecto). En este mismo contexto y tanto para grandes empresas como para pymes de la intermediación podría ser interesante saber cuál es el mecanismo por el que la información sobre sostenibilidad se convierte en conocimiento (capacidad de absorción) y cómo éste se puede transformar en la implementación de prácticas. Finalmente, también sería interesante desarrollar una línea de investigación futura que tuviera en cuenta la diferenciación entre perfiles señalada en este estudio y que permita ahondar en las circunstancias que permiten a los agentes reflejar su estilo de vida entre los consumidores finales y ver el papel que la proximidad que ofrecen las redes sociales y la tecnología pueden jugar al respecto. En un sector donde las innovaciones son rápidamente contrarrestadas por los imitadores y oportunistas sería oportuno analizar si el factor de legitimación vinculado a la proximidad con proveedores y consumidores permite orientar el proceso de reintermediación de este tipo de perfil de agentes para dificultar la imitación a través de la legitimidad percibida por los consumidores finales.

\section{BIBLIOGRAFÍA}

AGUIAR-QUINTANA, T., MORENO-GIL, S. y PICAZO-PERAL, P. (2016): «How could traditional travel agencies improve their competitiveness and survive? A qualitative study in Spain», Tourism Management Perspectives, $\mathrm{n}^{\circ}$ 20, pp. 98-108.

ALONSO-ALMEIDA, M. D. M., BAGUR-FEMENIAS, L., LLACH, J. y PERRAMON, J. (2015): «Sustainability in small tourist businesses: the link between initiatives and performance», Current Issues in Tourism, $\mathrm{n}^{\circ}$, pp. 1-20.

ANDAL-ANCION, A., CARTWRIGHT, P. y YIP, G. S. (2003): «The digital transformation of traditional business», MIT Sloan Management Review, no 44(4), pp. 34-41.

APPELMAN, J., GO, F., BUHALIS, D. y LAWS, E. (2001): «Transforming relationships between airlines and travel agencies: challenges for distribution and the regulatory framework», Tourism distribution channels: Practices, issues and transformations, $\mathrm{n}^{\circ}$, pp. 202-212.

BADDELEY, J., FONT, X., JAMAL, T. y DREDGE, D. (2011): «Barriers to tour operator sustainable supply chain management», Tourism recreation research, $\mathrm{n}^{\circ} 36(3)$, pp. 205-214.

BARTON, C., SCHLENKER, K. y EDWARDS, D. (2012): Understanding corporate social responsibility in small and medium tourism businesses. CAUTHE 2012: The new golden age of tourism and hospitality; Book 1; Proceedings of the 22nd Annual Conference, La Trobe University.

BÉDARD (2003): «Las agencias de viaje en la era del comercio electrónico», Cuadernos de Marketing. Publicaciones de la Organización Mundial del Turismo., $\mathrm{n}^{\circ}$ 1, pp. 71-90.

BERNE, C., GARCIA-GONZALEZ, M. y MUGICA, J. (2012): «How ICT shifts the power balance of tourism distribution channels», Tourism Management, $\mathrm{n}^{\circ}$ 33(1), pp. 205-214. 
BORJA, L. y GOMIS, J. M. (2009): El nuevo paradigma de la intermediación turística. Madrid, Ediciones Piramide.

BRAMWELL, B. y LANE, B. (1993): «Sustainable tourism: An evolving global approach», Journal of Sustainable Tourism, $\mathrm{n}^{\circ}$ 1(1), pp. 1-5.

BRØNN, P. S. y VIDAVER-COHEN, D. (2009): «Corporate motives for social initiative: legitimacy, sustainability, or the bottom line?», Journal of Business Ethics, $\mathrm{n}^{\circ}$ 87, pp. 91-109.

BUCKLEY, R. (2012): «Sustainable tourism: Research and reality», Annals of Tourism Research, no 39(2), pp. 528-546.

BUHALIS, D. (2003): eTourism: Information technology for strategic tourism management. Londres, Prentice Hall.

CARROLL, A. B. y SHABANA, K. M. (2010): «The business case for corporate social responsibility: a review of concepts, research and practice», International Journal of Management Reviews, $\mathrm{n}^{\circ}$ 12(1), pp. 85-105.

CLARKE, J. (1997): «A framework of approaches to sustainable tourism», Journal of Sustainable Tourism, $\mathrm{n}^{\circ}$ 5(3), pp. 224-233.

CLAVÉ, S. A. y REVERTÉ, F. G. (2007): A propósito del turismo: la construcción social del espacio turístico. Barcelona, Editorial UOC.

COLES, T., FENCLOVA, E. y DINAN, C. (2013): «Tourism and corporate social responsibility: A critical review and research agenda», Tourism Management Perspectives, $\mathrm{n}^{\mathrm{o}} 6$, pp. 122-141.

CONDON, L. (2004): «Sustainability and small to medium sized enterprises: how to engage them», Australian Journal of Environmental Education, $\mathrm{n}^{\circ}$ 20(1), pp. 57-67.

CURTIS, M. B., CONOVER, T. L. y CHUI, L. C. (2012): «A cross-cultural study of the influence of country of origin, justice, power distance, and gender on ethical decision making», Journal of International Accounting Research, $\mathrm{n}^{\circ} 11(1)$, pp. 5-34.

CHAN, E. y WONG, S. (2006): «Motivations for ISO14001 in the hotel industry», Tourism Management, $\mathrm{n}^{\circ} 27$, pp. 481-492.

CHIOU, W.-C., LIN, C.-C. y PERNG, C. (2011): «A strategic website evaluation of online travel agencies», Tourism Management, ${ }^{\circ}$ 32(6), pp. 1463-1473.

DAVIES, B. y DOWNWARD, P. (2007): «Exploring price and non-price decision making in the UK package tour industry: Insights from small-scale travel agents and tour operators», Tourism Management, $\mathrm{n}^{\circ}$ 28(5), pp. 1236-1261.

DEL ALCÁZAR, B. (2002): Los canales de distribución en el sector turístico. Madrid, ESIC Editorial.

DEO (2014): «Llistats d'establiments turístics.», Recuperado el 15/05/2014, de http:// empresaiocupacio.gencat.cat/ca/treb_ambits_actuacio/emo_turisme/emo_empreses_establiments_turistics/emo_registre_turisme_catalunya/emo_llistat_establiments_ turistics.

DODDS, R. y KUEHNEL, J. (2010): «CSR among Canadian mass tour operators: good awareness but little action», International Journal of Contemporary Hospitality Management, $\mathrm{n}^{\mathrm{o}}$ 22(2), pp. 221-244.

DWYER, L. (2005): «Relevance of triple bottom line reporting to achievement of sustainable tourism: A scoping study», Tourism Review International, $\mathrm{n}^{\circ}$ 9(1), pp. 79-938. 
FONT, X., GARAY, L. y JONES, S. (2014): «Sustainability motivations and practices in small tourism enterprises in European protected areas», Journal of Cleaner Production, $\mathrm{n}^{\mathrm{o}}$ In press, corrected proof.

FONT, X., GARAY, L. y JONES, S. (2016): «A social cognitive theory of sustainability empathy», Annals of Tourism Research, $\mathrm{n}^{\circ}$ 58, pp. 65-80.

FONT, X., GARAY, L. y JONES, S. (forthcoming): «Sustainability motivations and practices in small tourism enterprises», Journal of Cleaner Production, $\mathrm{n}^{\circ} 137, \mathrm{pp}$. 1439-1448.

FORD, R. C., WANG, Y. y VESTAL, A. (2012): «Power asymmetries in tourism distribution networks», Annals of Tourism Research, n 39(2), pp. 755-779.

GARAY, L. y FONT, X. (2012): «Doing good to do well? Corporate social responsibility reasons, practices and impacts in small and medium accommodation enterprises», International Journal of Hospitality Management, $\mathrm{n}^{\circ}$ 31(2), pp. 329-337.

GOMIS, J. M. y GONZÁLEZ-REVERTÉ, F. (2011): «Amadeus: global distribution system's new paradigm», en eTourism case studies. London, Butterworth Heinemann, pp. 399.

HARDY, A., BEETON, R. J. y PEARSON, L. (2002): «Sustainable tourism: An overview of the concept and its position in relation to conceptualisations of tourism», Journal of Sustainable Tourism, $\mathrm{n}^{\circ}$ 10(6), pp. 475-496.

HART, S. L. (1995): «A natural-resource-based view of the firm», Academy of management review, $\mathrm{n}^{\circ}$ 20(4), pp. 986-1014.

IDESCAT (2014): Anuari Estadistic de Catalunya 2013. Barcelona, Idescat. Institut d'Estadística de Catalunya. Generalitat de Catalunya.

INOUE, Y. y LEE, S. (2011): «Effects of different dimensions of corporate social responsibility on corporate financial performance in tourism-related industries», Tourism Management, $\mathrm{n}^{\mathrm{o}}$ 32(4), pp. 790-804.

JENKINS, H. (2009): «A 'business opportunity'model of corporate social responsibility for small-and medium-sized enterprises», Business Ethics: A European Review, ${ }^{\circ}$ 18(1), pp. 21-36.

KASIM, A. (2007): «Corporate Environmentalism in the Hotel Sector: Evidence of Drivers and Barriers in Penang, Malaysia», Journal of Sustainable Tourism, $\mathrm{n}^{\circ}$ 15(6), pp. 680-699.

KIM, D.-Y., LEHTO, X. Y. y MORRISON, A. M. (2007): «Gender differences in online travel information search: Implications for marketing communications on the internet», Tourism Management, $\mathrm{n}^{\circ}$ 28(2), pp. 423-433.

KOLLMUSS, A. y AGYEMAN, J. (2002): «Mind the gap: why do people act environmentally and what are the barriers to pro-environmental behavior?», Environmental education research, $\mathrm{n}^{\mathrm{o}} 8(3)$, pp. 239-260.

KRACHT, J. y WANG, Y. (2010): «Examining the tourism distribution channel: evolution and transformation», International Journal of Contemporary Hospitality Management, $\mathrm{n}^{\mathrm{o}} 22(5), \mathrm{pp} .736-757$.

LAW, R., LEUNG, K. y WONG, R. (2004): «The impact of the internet on travel agencies», International Journal of Contemporary Hospitality Management, $\mathrm{n}^{\circ} 16(2)$, pp. 100-107. 
LEE, S. y PARK, S.-Y. (2009): «Financial impacts of socially responsible activities on airline companies», Journal of Hospitality \& Tourism Research, $\mathrm{n}^{\circ}$.

MARKUS, H. R. y KITAYAMA, S. (1991): «Culture and the self: Implications for cognition, emotion, and motivation», Psychological review, n ${ }^{\circ}$ 98(2), pp. 224.

MIHALIC, T. (2014): «Sustainable-responsible tourism discourse-Towards 'responsustable'tourism», Journal of Cleaner Production, $\mathrm{n}^{\circ}$ 30, pp. 1e10.

MILLÁN, A. y ESTEBAN, Á. (2002): «Análisis de la competitividad. El caso de las agencias de viajes españolas», Annals of Tourism Research en español, $\mathrm{n}^{\circ} 4(1), \mathrm{pp} .211-238$.

MOLINA, J. F., CLAVER, E., PEREIRA, J. y TARÍ, J. J. (2009): «Environmental practices and firm performance: an empirical analysis in the Spanish hotel industry», Journal of Cleaner Production, $\mathrm{n}^{\circ}$ 17(5), pp. 516-524.

MORROW, D. y RONDINELLI, D. (2002): «Adopting Corporate Environmental Management Systems: Motivations and Results of ISO 14001 and EMAS Certification», European Management Journal, n ${ }^{\circ}$ 20(2), pp. 159-171.

NICOLAU, J. L. (2008): «Corporate Social Responsibility: Worth-Creating ctivities», Annals of Tourism Research, $\mathrm{n}^{\circ}$ 35(4), pp. 990-1006.

OROIAN, M. y GHERES, M. (2012): «Developing a risk management model in travel agencies activity: An empirical analysis», Tourism Management, $\mathrm{n}^{\mathrm{o}} 33(6)$, pp. 15981603.

PERRINI, F. (2006): «SMEs and CSR theory: evidence and implications from an Italian perspective», Journal of Business Ethics, nº 67(3), pp. 305-316.

PIFF, P. K., STANCATO, D. M., CÔTÉ, S., MENDOZA-DENTON, R. y KELTNER, D. (2012): «Higher social class predicts increased unethical behavior», Proceedings of the National Academy of Sciences, $\mathrm{n}^{\circ}$ 109(11), pp. 4086-4091.

POON, A. (2001): «The future of travel agents», Travel \& Tourism Analyst, $\mathrm{n}^{\circ}$ (3), pp. 57-80.

RAVIV, C., BECKEN, S. y HUGHEY, K. F. (2013): «Exploring Values, Drivers, and Barriers as Antecedents of Implementing Responsible Tourism», Journal of Hospitality \& Tourism Research, $\mathrm{n}^{\circ}$.

RUHANEN, L., WEILER, B., MOYLE, B. D. y MCLENNAN, C.-L. J. (2015): «Trends and patterns in sustainable tourism research: a 25-year bibliometric analysis», Journal of Sustainable Tourism, no 23(4), pp. 517-535.

SALZMANN, O., IONESCU-SOMERS, A. y STEGER, U. (2005): «The business case for corporate sustainability:: literature review and research options», European Management Journal, $\mathrm{n}^{\mathrm{o}}$ 23(1), pp. 27-36.

SAMPAIO, A. R., THOMAS, R. y FONT, X. (2012): «Why are some engaged and not others? Explaining environmental engagement among small firms in tourism», International Journal of Tourism Research, $\mathrm{n}^{\circ}$ 14(3), pp. 235-249.

SHANE, S., LOCKE, E. y COLLINS, C. (2003): «Entrepreneurial motivation», Human resource management review, $\mathrm{n}^{\circ} 13(2)$, pp. 257-279.

SHELDON, P. J. y PARK, S.-Y. (2010): «An exploratory study of corporate social responsibility in the US travel industry», Journal of Travel Research, $\mathrm{n}^{\circ}$, pp. 0047287510371230 . 
SIGALA, M. (2008): «A supply chain management approach for investigating the role of tour operators on sustainable tourism: the case of TUI», Journal of Cleaner Production, $\mathrm{n}^{\circ} 16(15)$, pp. 1589-1599.

STANDING, C., TANG-TAYE, J.-P. y BOYER, M. (2014): «The Impact of the Internet in Travel and Tourism: A Research Review 2001-2010», Journal of Travel \& Tourism Marketing, $\mathrm{n}^{\mathrm{O}}$ 31(1), pp. 82-113.

SU, X., WANG, H. y WEN, T. (2013): «Profit, Responsibility, and the Moral Economy of Tourism», Annals of Tourism Research, $\mathrm{n}^{\circ}$ 43, pp. 231-250.

THAPA, B. y BEST, M. N. (2013): «Motives, Facilitators and Constraints of Environmental Management in the Caribbean Accommodations Sector», Journal of Cleaner Production, $\mathrm{n}^{\circ}$ 52(1), pp. 165-175.

TZSCHENTKE, N., KIRK, D. y LYNCH, P. (2008): «Ahead of their time? Barriers to action in green tourism firms», The Service Industries Journal, ${ }^{\circ}$ 28(2), pp. 167-178.

TZSCHENTKE, N., KIRK, D. y LYNCH, P. A. (2008): «Ahead of their time? Barriers to action in green tourism firms», Service Industries Journal, n 28(2), pp. 167-178.

VALLESPÍN ARÁN, M. y MOLINILLO, S. (2014): «El futuro de la intermediación en el sector turístico», Revista de análisis turístico, $\mathrm{n}^{\circ}$ (17), pp. 13-25.

WALIGO, V. M., CLARKE, J. y HAWKINS, R. (2013): «Implementing sustainable tourism: a multi-stakeholder involvement management framework», Tourism Management, $\mathrm{n}^{\mathrm{o}} 36$, pp. 342-353.

WCED, U. (1987): «Our common future», World Commission on Environment and DevelopmentOxford University Press, $\mathrm{n}^{\circ}$.

WEAVER, D. B. (2013): «Asymmetrical dialectics of sustainable tourism: Toward enlightened mass tourism», Journal of Travel Research, $\mathrm{n}^{\circ}$, pp. 0047287513491335.

WORTHINGTON, I., RAM, M. y JONES, T. (2006): “'Giving something back”: a study of corporate social responsibility in UK South Asian small enterprises», Business Ethics: A European Review, no 15(1), pp. 95-108. 